OPEN ACCESS

Edited by:

Fiorenzo Conti,

Marche Polytechnic University, Italy

Reviewed by:

Heinrich S. Gompf,

University of Massachusetts Medical

School, United States

Michael Telias,

University of California, Berkeley,

United States

*Correspondence:

Corinna Giorgi

c.giorgi@ebri.it

Silvia Marinelli

s.marinelli@ebri.it

Specialty section:

This article was submitted to Neuroplasticity and Development,

a section of the journal

Frontiers in Molecular Neuroscience

Received: 01 April 2021

Accepted: 18 May 2021

Published: 15 June 2021

Citation:

Giorgi C and Marinelli S (2021) Roles and Transcriptional Responses of Inhibitory Neurons in Learning and

Memory.

Front. Mol. Neurosci. 14:689952. doi: 10.3389/fnmol.2021.689952

\section{Roles and Transcriptional Responses of Inhibitory Neurons in Learning and Memory}

\author{
Corinna Giorgi ${ }^{1,2 *}$ and Silvia Marinelli ${ }^{2 *}$ \\ ${ }^{1}$ CNR, Institute of Molecular Biology and Pathology, Rome, Italy, ${ }^{2}$ European Brain Research Institute (EBRI), Fondazione Rita \\ Levi-Montalcini, Rome, Italy
}

Increasing evidence supports a model whereby memories are encoded by sparse ensembles of neurons called engrams, activated during memory encoding and reactivated upon recall. An engram consists of a network of cells that undergo longlasting modifications of their transcriptional programs and connectivity. Ground-breaking advancements in this field have been made possible by the creative exploitation of the characteristic transcriptional responses of neurons to activity, allowing both engram labeling and manipulation. Nevertheless, numerous aspects of engram celltype composition and function remain to be addressed. As recent transcriptomic studies have revealed, memory encoding induces persistent transcriptional and functional changes in a plethora of neuronal subtypes and non-neuronal cells, including glutamatergic excitatory neurons, GABAergic inhibitory neurons, and glia cells. Dissecting the contribution of these different cellular classes to memory engram formation and activity is quite a challenging yet essential endeavor. In this review, we focus on the role played by the GABAergic inhibitory component of the engram through two complementary lenses. On one hand, we report on available physiological evidence addressing the involvement of inhibitory neurons to different stages of memory formation, consolidation, storage and recall. On the other, we capitalize on a growing number of transcriptomic studies that profile the transcriptional response of inhibitory neurons to activity, revealing important clues on their potential involvement in learning and memory processes. The picture that emerges suggests that inhibitory neurons are an essential component of the engram, likely involved in engram allocation, in tuning engram excitation and in storing the memory trace.

Keywords: interneurons, memory, engram, IEGs, GABA, activity-dependent transcription, plasticity

\section{A RECAP ON ENGRAMS}

Higher cognitive functions such as learning, memory and processing of sensory perceptions, are strictly dependent on the correct flow of information within neuronal circuits made of both inhibitory and excitatory neurons. Further, it is the proper balance between excitation and inhibition that is particularly important for the correct execution of these brain functions.

Learning is the process during which an individual acquires a new information, thus representing the early phase of the formation or encoding of a memory. Memory, on the other 
hand, may be defined as the process that allows consolidation, storage and recall of the acquired information (Sherwood, 2015). The physical substrate of memory is assumed to be located in the so-called engram, a sparse ensemble of neurons that has the following properties: (i) is activated by a learning experience; (ii) has persistent structural and/or chemical modifications that affect their excitability and circuitry; (iii) is reactivated upon presentation of those same stimuli that triggered learning acquisition, resulting in memory retrieval (Reijmers et al., 2007; Liu et al., 2012; Josselyn et al., 2015; Josselyn and Tonegawa, 2020). Engram cells are thought to be connected into an "engram cell ensemble," that once consolidated constitutes the cellular substrate of memory (Poo et al., 2016; Josselyn and Tonegawa, 2020). The current understanding is that neurons forming an engram ensemble are connected by strengthened synapses (Ryan et al., 2015), providing a conceptual link with the synaptic plasticity paradigm for learning and memory (Morris et al., 2003). This posits that the cellular correlate of memory-driven behavior is represented by the experimental phenomena of synaptic plasticity, characterized by enduring changes of synaptic strength such as long-term potentiation or depression (LTP or LTD) (Abraham et al., 2019). Accordingly, the encoding of information relies on the synaptic strengthening of both existing (Liao et al., 1995; Le Bé and Markram, 2006) and newly formed neuronal connections (Matsuzaki et al., 2004), along with increased excitability of ensemble neurons (Disterhoft et al., 1986; Oh and Disterhoft, 2015; Kim et al., 2016; Lisman et al., 2018).

How are active neurons allocated into an engram ensemble? According to Josselyn et al. (2015), during the encoding phase, active neurons in a given brain region compete against each other for allocation to an engram ensemble. Indeed, not all activated neurons will become engram cells supporting a particular memory: only a subset will be selected as engram ensemble neurons (memory allocation) based on their intrinsic excitability and increased synaptic strength (Zhou et al., 2009; Nonaka et al., 2014; Yiu et al., 2014; Gouty-Colomer et al., 2016; Park et al., 2016; Sehgal et al., 2018). Neurons with relatively increased intrinsic excitability, at the time of learning, win this competition to become engram cells (Han et al., 2007; Yiu et al., 2014). Several lines of evidence suggest that an additional mechanism shaping engram size involves inhibitory neurons (Holtmaat and Caroni, 2016; Morrison et al., 2016; Stefanelli et al., 2016; Josselyn and Tonegawa, 2020). Following excitatory plasticity, the potentiation of GABAergic activity may directly control the excitability of principal neurons by either disinhibition mechanisms (Donato et al., 2013; Cummings and Clem, 2020), or by lateral inhibition (Morrison et al., 2016; Stefanelli et al., 2016). While in the first, the enhancement of GABAergic neurotransmission causes an increase of projecting neurons excitability by inhibiting a relay interneuron, in the latter, the boost of inhibition triggered by activation of principal cells leads to inhibition of neighboring projecting neurons.

The size of an engram within a given brain region (that is, the number of engram cells in an ensemble) is stable and remains constant as memory strength increases (Han et al., 2007). Hence, memory strength does not affect engram size. Rather, an increased number and size of spines between engram cells underlies a stronger memory (Choi et al., 2018).

Once the engram ensemble is allocated, what makes it long-lasting? Increasing evidence suggests that memory persistence relies on structural and epigenetic modifications of engram neurons, which ultimately govern their connectivity and excitability (Frey et al., 1996; Kandel, 2001; Alberini and Kandel, 2014; Duke et al., 2017; Clayton et al., 2020). As described in a subsequent section of this review, these modifications are initiated at the very first stage of memory acquisition, whereby behavioral experiences trigger transcription and translation of activity-dependent genes. Expression of these immediate early genes (IEGs), including Arc, Fos, and Npas4, in turn activates diverse molecular programs which fundamentally modify synaptic strength, connectivity and epigenome of the activated ensemble. The persistence of such modifications is also dependent on activity-dependent expression of IEGs. Additionally, protein synthesis is crucial for the consolidation of a memory and it is via de novo translation of synaptic plasticity proteins that chemical and structural modifications (i.e., generation and deletion of dendritic spines, increase of synaptic strength and connectivity) become persistent (Bekinschtein et al., 2007; Matsuo et al., 2008; Yang et al., 2009; Sanders et al., 2012; Morris, 2013; Nonaka et al., 2014). Notably, with the exception of synaptic connectivity, these enduring changes are present in consolidated engram cells of a selected cellular memory trace (Ryan et al., 2015), but are lost in models of retrograde amnesia. For instance, in murine models of Alzheimer's disease, or upon protein synthesis inhibition, engram neurons become "amnesic" as the increased spine density, enhanced synaptic strength and memory-guided behavior are lost. However, the connectivity between these neurons still persists and allows artificial memory retrieval (Ryan et al., 2015; Roy et al., 2016). Indeed, in amnesic animals, the natural memory recall is disrupted and only artificial (optogenetic) activation of "amnesic" but still connected engram cells, succeeds in memory retrieval. Therefore, while the long lasting potentiation of synapses is a prerequisite for encoding, formation and retrieval of memory traces (Garner et al., 2012; Ramirez et al., 2013; Nabavi et al., 2014; Lopez et al., 2015; Ryan et al., 2015), it may be dispensable for their storage once they are consolidated (Tonegawa et al., 2015).

The "engram paradigm" as discussed above, highlights neurons as the main component of the engram ensemble. Zooming in from this cellular level, to the finer spatial scale of the synapses, memory engrams can also be conceived as synaptic ensembles (Hayashi-Takagi et al., 2015; Poo et al., 2016; Gobbo et al., 2017), as indeed spines and synapses are considered the building blocks of memory (Roberts et al., 2010; Lai et al., 2012; Moczulska et al., 2013; Choi et al., 2018). Conversely, zooming out from the cellular level to the larger scale of neuronal networks, sets the focus on the interplay between inhibitory and excitatory neurons in engram ensembles.

In this regard, a major unresolved issue revolves around the cellular composition of engram ensembles and how different neuronal subtypes contribute to its formation and function. Current strategies adopted to map and manipulate memory engrams do not discriminate between the excitatory or inhibitory 
identity of the cells involved. Rather, independent evidence exists that engram includes both an inhibitory and an excitatory component. While principal neurons are often the target of memory trace studies, the inhibitory component of the engram is often overlooked. Nonetheless, increasing evidence shows that GABAergic inhibitory neurons play an integral role in engram formation, storage, and retrieval (Artinian and Lacaille, 2018; Lucas and Clem, 2018; Lamsa and Lau, 2019). Further, understanding how excitation and inhibition cooperate to form and maintain memory engrams is of pivotal importance. Hence, in this article, we review available evidence on the involvement of GABAergic interneurons in memory engram formation and function, with a focus on their emerging role in memory maintenance. In addition, we provide an overview of GABAergic interneuron transcriptional profiles in response to experience, extrapolating important hints on their contribution to learning and memory processes.

\section{RECRUITMENT AND FUNCTIONS OF INHIBITORY INTERNEURONS IN DISTINCT MEMORY PHASES}

Over the last decade, a growing number of experimental and theoretical studies unveiled that the cooperation between GABAergic and glutamatergic neurons is fundamental to the shaping of different memory stages, including the encoding, consolidation, storage and recall (Vogels et al., 2011; Lucas and Clem, 2018). Hereafter, we will provide an overview of the role played by inhibitory neurons in distinct memory stages, detailing the mechanisms involved.

One route through which inhibitory neurons may be recruited to an engram is through signaling of neuromodulatory molecules. In an associative memory task, it has been shown that upon unconditioned aversive stimuli, acetylcholine elicits activation of somatostatin positive (SOM+) dendritic targeting-interneurons, promoting fear learning. This increase in GABAergic activity is required for memory formation, through inhibition of CA1 principal cells that received an enhanced excitation from the entorhinal cortex (Lovett-Barron et al., 2014). Another possible signaling that may contribute to GABAergic neuron recruitment into the engram ensemble is the dopaminergic one (Karunakaran et al., 2016). In this study, the authors found that activation of dopamine receptors is necessary to maintain high levels of parvalbumin positive $(\mathrm{PV}+)$ basket cell plasticity and ensures memory consolidation. Further, secretion of the brain derived neurotrophic factor (BDNF), elicited by Npas4 expression in pyramidal neurons of mice exposed to an enriched environment, increases the number of somatic inhibitory inputs onto principal cells, while their dendritic inhibition is decreased (Bloodgood et al., 2013). This redistribution of inhibitory circuitry may underlie fear memory formation, since the knockdown of Npas4 in the lateral amygdala, impaired memory fear encoding and retention (Ploski et al., 2011).

In addition to neuromodulatory molecules, homeostatic mechanisms may represent another potential route to engage GABAergic neurons in engram allocation. Indeed, both preclinical and clinical evidence revealed that an increase in inhibitory neuron activity is triggered in response to hyperexcitability of principal neurons and is required for proper brain functions (Barron et al., 2016; Fernandes and Carvalho, 2016; Chiu et al., 2019).

In the formation and consolidation of a memory trace, the potentiation of excitatory synapses among engram neurons, must be homeostatically compensated to prevent overexcitation of the neural network. Indeed, an increase in inhibitory synaptic plasticity can integrate excitatory plasticity in a memory network (Vogels et al., 2011). Stefanelli et al. (2016) observed that optogenetic stimulation of sparse granule cells induces an increased GABAergic response onto the dendrites of surrounding granule cells. This lateral inhibition of non-active principal cells relies on $\mathrm{SOM}+$ interneurons activation and represents a mechanism to control engram size during formation of a memory trace. Likewise, in the lateral amygdala, a sustained activity of $\mathrm{PV}+$ basket cells is necessary to shape engrams during the encoding of new information (Morrison et al., 2016). Overall, these studies point to a potentiation of GABAergic neurotransmission in controlling the engram size, during memory allocation.

GABAergic neurons can modulate activity of principal cells directly, or indirectly by targeting other interneurons (relay interneurons), which in turn contact excitatory neurons. This cellular arrangement, referred to as disinhibition, allows GABAergic cells to indirectly control the inhibitory tone onto glutamatergic neurons. Both direct inhibition and disinhibition play important roles in memory formation and recall. For instance, disinhibition is a common mechanism utilized to release the activity of excitatory engram cells in different phases of associative memory (Letzkus et al., 2015; Artinian and Lacaille, 2018).

During fear learning, exposure to an auditory cue induces excitation of $\mathrm{PV}+$ interneurons which disinhibit amygdala principal cells by inhibiting SOM+ interneurons, with the final outcome of an enhanced auditory response and learning (Wolff et al., 2014). Another di-synaptic inhibitory circuit has been identified in the dorsolateral striatum of mice, in which $\mathrm{PV}+$ interneurons control the output of medium spine neurons via neurogliaform interneurons, thereby improving learning (Lee et al., 2018). Subsequent independent studies observed an increased activity of cortical SOM+ neurons during both social fear expression and fear memory acquisition. This enhancement was necessary for both social fear and fear memory-guided behaviors. In particular, social fear and freezing behavior were dependent on SOM+ interneuron activation, which inhibits $\mathrm{PV}+$ cells, ultimately disinhibiting cortical pyramidal neurons (Xu et al., 2019; Cummings and Clem, 2020). Thus, disynaptic disinhibition of pyramidal neurons, due to activation of upstream GABAergic cells, clearly contributes to memory encoding and recall. However, multiple studies converge in indicating that memory encoding is also accompanied by an increase in inhibitory drive directly onto activated pyramidal neurons. For instance, recent evidence finely identifies mechanisms regulating the perisomatic inhibitory plasticity onto activated CA1 pyramidal neurons (Yap et al., 2021). The 
authors observed that an enhanced and persistent perisomatic inhibition by $\mathrm{PV}+$ interneurons onto Fos-activated pyramidal cells was counterbalanced by a weaker perisomatic inhibition by cholecystokinin expressing basket cells (CCKBC) (Yap et al., 2021). This bidirectional inhibition was regulated by Fosinduced Scg2 expression, whose suppression was shown to disrupt hippocampal network oscillations. The authors argue that Fos-driven reorganization of inhibitory inputs from $\mathrm{PV}+$ and CCKBC may represent a mechanism for selecting a cell's eligibility to take part in the encoding and recall phases of memory processes. Viceversa, activity-dependent expression of the IEG transcription factor NPAS4 in a subset of pyramidal neurons, selectively enhanced somatic inhibition mediated by CCKBCs, but not by PVBC (Hartzell et al., 2018).

Altogether, these studies emphasize the strategic functions of the inhibitory component of the engram in the early stages of memory formation.

A critical contribution of enhanced GABAergic activity was also observed in contextual fear memory consolidation. Indeed, pharmacogenetic inhibition of PV interneurons, in either CA1 or anterior cingulate cortex, disrupted brain oscillations along with memory strengthening (Ognjanovski et al., 2017; Xia et al., 2017). Additional evidence from CA3 and motor cortex points to a PV-network plasticity mechanism, induced by modulating VIP (vasoactive intestinal peptide)-PV microcircuits, as a requisite for long-term memory consolidation, retrieval and learning (Donato et al., 2013). In this study, motor learning tasks increased $\mathrm{VIP}+$ inhibitory inputs onto PV + cells. Upon completion of memory acquisition, excitatory inputs onto PV+ cells increased, leading to a network state dominated by inhibition. Hence, these studies highlight the potentiation of GABAergic activity as a process essential to memory maintenance.

Aside from the indirect di-synaptic disinhibition of pyramidal neurons, a straightforward reduction of inhibitory inputs, or of GABA levels, underlies the process of memory reinstatement after extinction-guided behavior and of memory recall (Courtin et al., 2014; Barron et al., 2016; Vallentin et al., 2016). During fear behavior, PV+ basket cell inhibition, achieved via optogenetic approaches or upon presentation of a neutral conditioned stimulus, directly increased the activity of cortical projecting neurons and reinstated the fear response after fear conditioning and extinction (Courtin et al., 2014). The inhibition of PV+ interneurons, as that of $\mathrm{SOM}+$ cells, represents an important mechanism for gating fear learning. Aversive stimuli-induced interneuron inhibition led to an enhancement of the fear response by disinhibiting the entire somatodendritic compartment of principal cells, whose increased excitability is essential for memory trace acquisition (Wolff et al., 2014).

\section{THE INHIBITORY COMPONENT OF THE ENGRAM IN THE LATENT PHASE OF MEMORY STORAGE}

As initially reported by Richard Semon in his description of the engram (Schacter, 2001), a consolidated memory trace lies in a dormant state before being reactivated by recall and memory retrieval.

So, what is keeping a memory latent in this storage phase? At the anatomical level, it is evident that reorganization of specific brain circuitry (i.e., hippocampus, cortex, amygdala) underlies memory storage in a time dependent manner (Bontempi et al., 1999; Frankland et al., 2004; Tonegawa et al., 2018). Nonetheless, the molecular mechanisms that support the retention of an engram are still unclear. The ongoing connections between engram cells, formed during learning and observed in silent engrams, may represent a way in which memory is retained (Tonegawa et al., 2015; Roy et al., 2017; Abraham et al., 2019). Unlike the normal and dormant engram cells, silent neuronal ensembles cannot be retrieved by natural stimuli and exhibit less increased synaptic strength and spine density (reviewed in Josselyn and Tonegawa, 2020). Additional mechanisms might be epigenetic modifications (Halder et al., 2016; Pearce et al., 2017; Perry et al., 2017; Clayton et al., 2020), wherein DNA methylation controls the maintenance of long-term memory changes in engram cells. Further, homeostatic plasticity processes (Turrigiano, 2012; Sprekeler, 2017; Goel and Dickman, 2021), such as synaptic scaling and potentiation of inhibition, could also contribute to memory retention. The potentiation of inhibition acts in response to an increased activity of postsynaptic projecting neurons to rebalance the hyperexcited neural circuitry (Marinelli et al., 2009; Lourenço et al., 2014; Chiu et al., 2018, 2019; Mendez et al., 2018). Modeling the activity pattern of spike rates, it emerged that the inhibitory connectivity, despite being equipped with a smaller number of synapses, determined these spike networks and was more effective in storing memory patterns than the excitatory connectivity. The rewiring of excitatory-toexcitatory connections did not have strong effects on the activity pattern of the spike network model, while the rewiring of the inhibitory-to-inhibitory connections led to a loss of memory recall. As a consequence, inhibitory circuits could have the potential to control the stability of a memory for a long time (Mongillo et al., 2018). Moreover, human studies revealed that an increase in cortical GABA concentration could represent a mechanism to keep memories latent. Indeed, a reduction in GABA levels, by anodal transcranial direct current stimulation, allowed a recall of dormant memories, suggesting that balanced excitatory-inhibitory neuronal ensembles are pivotal for the storage of memory traces in a latent state (Barron et al., 2016).

Earlier evidence indicated that during the initial stages of learning, pyramidal neurons of rabbit hippocampi showed increased spiking activity which decreased in later stages of learning, when the firing rate of theta cells (presumably interneurons) was enhanced (McEchron and Disterhoft, 1997). Similarly, a long-term enhancement of inhibitory synaptic transmission was recorded in mouse hippocampi, 5 days after an olfactory learning task (Ghosh et al., 2015). In keeping with these studies, anatomical structural plasticity evidence indicates an experience-dependent increase in feed-forward inhibition, paralleled by growth of inhibitory synapses onto CA3 pyramidal neurons for days after learning (Ruediger et al., 2011). This increased feedforward inhibition was required for memory precision and storage: photostimulation of 
$\mathrm{PV}+$ interneurons promotes the enhancement of feedforward inhibition, maintaining the fear memory engram over time (Guo et al., 2018).

A recent paper underscores the importance of inhibitory synaptic plasticity occurring in parallel with excitatory synaptic plasticity, as a critical early step in preserving memory (Davenport et al., 2021). By optically controlling the translocation of alfa5-GABA $A$ receptors from extrasynaptic to synaptic inhibitory synapses, upon high-frequency stimulation of the excitatory input, a hidden and prolonged synaptic inhibition was revealed, that prevents accumulation of excitatory LTP. This form of metaplasticity, arising from the long-term changes of synaptic strength, may stabilize LTP/memory consolidation and may behave as an enduring event. Intriguingly, the inhibitory component of memory engrams may exert its functions through this form of plasticity.

All these lines of evidence suggest that potentiated inhibitory synapses onto principal neurons may be a necessary and sufficient condition for retaining engram cell ensembles in a dormant state until their reactivation upon memory recall.

The above reported studies prompt further investigations to elucidate if ensembles of active GABAergic neurons behave as the inhibitory component of engrams along the sequential phases of learning and memory. In particular, it remains to be experimentally ascertained whether GABAergic interneurons operate in memory processes as an integral component of the engram (e.g., allocated during learning, activated during storage and modulated during recall), or as accessory cells that merely gate the excitation of glutamatergic engram neurons.

Transcriptomic analyses are an emerging field of study that can significantly contribute to addressing these issues. As detailed in the following section, this approach has been utilized to interrogate the transcriptional response of specific neuronal subclasses to experience-induced activation or reactivation, unveiling new clues as to the contribution of inhibitory neurons to memory formation and recall.

\section{ACTIVITY-DEPENDENT TRANSCRIPTION: A CLOSER LOOK AT THE INHIBITORY COMPONENT OF MEMORY ENGRAMS}

\section{Engram Mapping Strategies With Neuronal-Subtype Specificity}

Critical advancements in memory engram mapping and manipulation have been enabled only recently by the ingenious combination of cutting-edge genetic tools exploiting the molecular cascades triggered by experience within engram neurons (Han et al., 2009; Liu et al., 2012). A common strategy adopted to label activated neuronal ensembles takes advantage of enhancer and promoter sequences of activity-dependent genes whose transcription is activated upon plasticity-inducing stimuli (Kawashima et al., 2014; DeNardo and Luo, 2017;
Josselyn and Tonegawa, 2020). The promoters most widely utilized to aid neuronal activity mapping are those of the Fos, Erg1, and Arc/Arg3.1 genes (Schilling et al., 1991; Waltereit et al., 2001; Kawashima et al., 2009; Pintchovski et al., 2009; Guenthner et al., 2013; DeNardo and Luo, 2017; Sauvage et al., 2019). Alternatively, the recently developed RAM system allows for active ensemble neuron labeling thanks to a synthetic promoter which combines the binding sites for the activity-dependent transcription factors Fos and Npas4 (Sørensen et al., 2016). As detailed below, however, expression of these well-known activitydependent genes is not limited to one neuronal subtype, rather they appear to be induced in both excitatory and inhibitory neurons (Spiegel et al., 2014; Hrvatin et al., 2018). This implies that the engram labeling and manipulation strategies achieved, for example, through Fos-driven expression of optogenetic or chemiogenetic tools (DeNardo and Luo, 2017) are not restricted to a neuronal subtype. Rather, they perturb both GABAergic and glutamatergic components of the activated ensemble, yielding results that cannot be ascribed to either neuronal subtype.

Genetic strategies that can enable further restriction of engram manipulation to a specific neuronal subtype are now emerging, mainly combining subtype-specific CRE-driver mouse lines with a CRE-dependent expression of activity-driven effector genes. A promising example of this tactic is the CRAM construct, a CRE-dependent modification of the RAM system, which allows to investigate sub-populations of engram ensembles in a neuronal-subtype specific manner (Sørensen et al., 2016).

An alternative avenue to gain subtype specificity in engram manipulation could otherwise stem from the identification and exploitation of activity-dependent genes that are expressed only in a specific neuronal-subtype. While this characterization is still at its infancy, a growing number of studies investigating activity-dependent transcription in a cell-type specific manner, or at a single-cell level, are starting to emerge and represent an untapped resource. In the quest to investigate the functional contribution of GABAergic neurons to memory formation, consolidation and storage it is therefore important to take into account the activity-dependent transcriptional profiles of GABAergic neurons as a whole and of its numerous subclasses. Additionally, and as it will be discussed below, activity-dependent transcription critically contributes to the reorganization of synaptic connectivity within engram cells in response to activity. Thus, investigating the transcriptional responses of interneurons is a key step toward a deeper understanding of the mechanisms underlying the formation and functional responses of the inhibitory component of memory engrams. Hence, in the following sections, we will provide an overview of recent advancements in the field of celltype specific activity-dependent transcription with a focus on GABAergic interneurons.

\section{Molecular Mechanisms Underlying Activity-Dependent Transcription}

For many years, it has been broadly accepted that changes in gene expression patterns provide an essential contribution to the long-term modification of synaptic strength and to 
memory consolidation (Goelet et al., 1986; Nguyen et al., 1994; Alberini and Kandel, 2014; Minatohara et al., 2016). The current view is that, in response to experience, neuronal activity triggers both transcription of activity-dependent genes and chromatin modifications (Duke et al., 2017; Yap and Greenberg, 2018; Fernandez-Albert et al., 2019; Clayton et al., 2020), resulting in a substantially altered epigenomic and transcriptomic profile of that neuron. The initial transcriptional response is transient but leads to two major waves of activitydependent gene expression with long-term effects. The first highly transient wave of transcriptional induction includes a set of genes, referred to as Immediate Early Genes (IEG), that encode several transcription factors (i.e., Fos, Npas4, Erg1-3) or a sub-set of plasticity-effector proteins (i.e., Arc, Amigo3) (Tyssowski et al., 2018; Yap and Greenberg, 2018). Sustained activity and these IEG transcription factors, in turn, elicit the second wave of late-response genes (LRG) expression. These include epigenetic modulators, secreted factors and neuromodulators (Mardinly et al., 2016; Su et al., 2017; Hrvatin et al., 2018; Jaeger et al., 2018), responsible for translating the transcriptional response into a functional one and promoting cell-type specific cellular and synaptic changes at the circuit level.

Importantly, while IEG transcriptional activation is independent from new protein synthesis, LRG activation is dependent on protein translation particularly of mRNAs encoding IEG transcription factors (Tyssowski et al., 2018). This distinction is important as it highlights the complex strategy utilized by neuronal cells to respond in a timely manner to activity without depending on the time-consuming protein translation process. Two molecular mechanisms have been described that contribute to ensure the fast kinetics of IEG transcription. Firstly, membrane depolarization triggered by neuronal activity induces calcium entry trough NMDA receptors and L-type calcium channels, initiating signaling cascades, primarily those involving the ERK/MAPK Kinases, which lead to the activation of pre-existing transcription factors such as the cyclic adenosine monophosphate (cAMP)-responsive element binding protein (CREB), the myocyte enhancer factor 2 (MEF2), and serum response factor (SRF) (Kawashima et al., 2009; Pulimood et al., 2017; Tyssowski et al., 2018). These transcription factors, in turn, activate transcription of almost all known IEGs. A second mechanism contributing to a rapid induction of IEG genes relies on their promoters being predisposed to swift activation by recruitment of Poised PolII complexes and open chromatin states (Saha et al., 2011; Tyssowski et al., 2018).

The molecular cascades underlying activity-dependent transcription are common to most neuronal cells analyzed. However, the resulting pool of mRNAs induced in different areas of the brain and in different cell types is strikingly specific. Upon learning, distinct brain regions display highly divergent transcriptional profiles, as best exemplified in recent transcriptomic analyses performed from dorsal hippocampi and prelimbic cortices of mice subjected to inhibitory avoidance learning (Katzman et al., 2021). As detailed below, great heterogeneity also characterizes the activity-dependent transcriptional profiles of different neuronal subtypes in the same brain region, reflecting and facilitating their functional specialization.

\section{Subtype Specificity of Activity-Dependent Transcription}

Activity-dependent gene expression has been investigated for decades; however, early studies have been limited in resolution and detection sensibility as they were based on pharmacologically induced generalized activation applied on cultured neurons or bulk tissues (Loebrich and Nedivi, 2009).

The first study to pioneer a comprehensive analysis of neuronal subtype specificity in activity-driven transcriptomes was carried out by Spiegel et al. (2014), comparing the transcriptional response of cortical inhibitory neurons to that of excitatory neurons both in vitro and in vivo. Microarray analyses revealed that, at $1 \mathrm{~h}$ of $\mathrm{KCl}$ depolarization, cultures enriched of excitatory neurons or of GABAergic neurons express a highly overlapping set of IEGs. In particular, all but one of the transcriptional regulators acutely induced by depolarization in GABAergic cultures were found also induced in excitatory neurons. These common IEGs include Fos, FosB, Egr1-3, Nr4a1, and Npas4. Strikingly, the well-studied plasticity-effector protein Arc, commonly considered a primarily glutamatergic IEG (Vazdarjanova et al., 2006; Morrison et al., 2016; Nikolaienko et al., 2018), resulted among the IEG induced in both cortical interneurons and excitatory neurons in culture. However, substantial differences in the transcriptional profiles of inhibitory and excitatory neurons clearly emerge when the authors investigate the second wave of LRG induction. At $6 \mathrm{~h}$ from depolarization, they found only a minimal overlap (25\% of LRG transcripts) in the transcriptional responses of inhibitory and excitatory neuronal cultures. With this approach, for the first time, hundreds of late response genes specifically induced in activated interneurons have been identified, including Cacng5, Igf1, Pnoc, Pthlh and E530001K10RIK (Table 1). Surprisingly, this analysis also revealed that several well-known LRGs, such as Bdnf, Homer1 and Cpg15/Nrn1, are selectively induced in excitatory neurons and not in inhibitory neurons. These important findings were also corroborated in vivo, through identification of ribosome-associated transcripts within cortical GABAergic or glutamatergic neurons in Ribo-Tag mice (Sanz et al., 2009) undergoing visual stimulation. Hence, in this study, both in vitro depolarization and in vivo visual stimulation induced a similar set of immediate early genes in inhibitory and excitatory neurons. However, depending on the neuronal subtype, this same set of transcription factors appears to drive distinct downstream LRG transcriptional profiles, possibly as a consequence of differential epigenetic modifications and accessibility of their target enhancer and promoter elements in each neuronal subtype (Yap and Greenberg, 2018). To further investigate this important finding, Spiegel et al. (2014) proceeded to assess the role of the IEG transcription factor Npas4 in driving LRG transcriptional profiles in excitatory vs. inhibitory neurons. Npas4 had been previously suggested to regulate the inhibitoryexcitatory balance of neural circuits and was shown to promote perisomatic inhibition of excitatory neurons (Lin et al., 2008; 
TABLE 1 | Examples of IEGs and LRGs induced in inhibitory neurons.

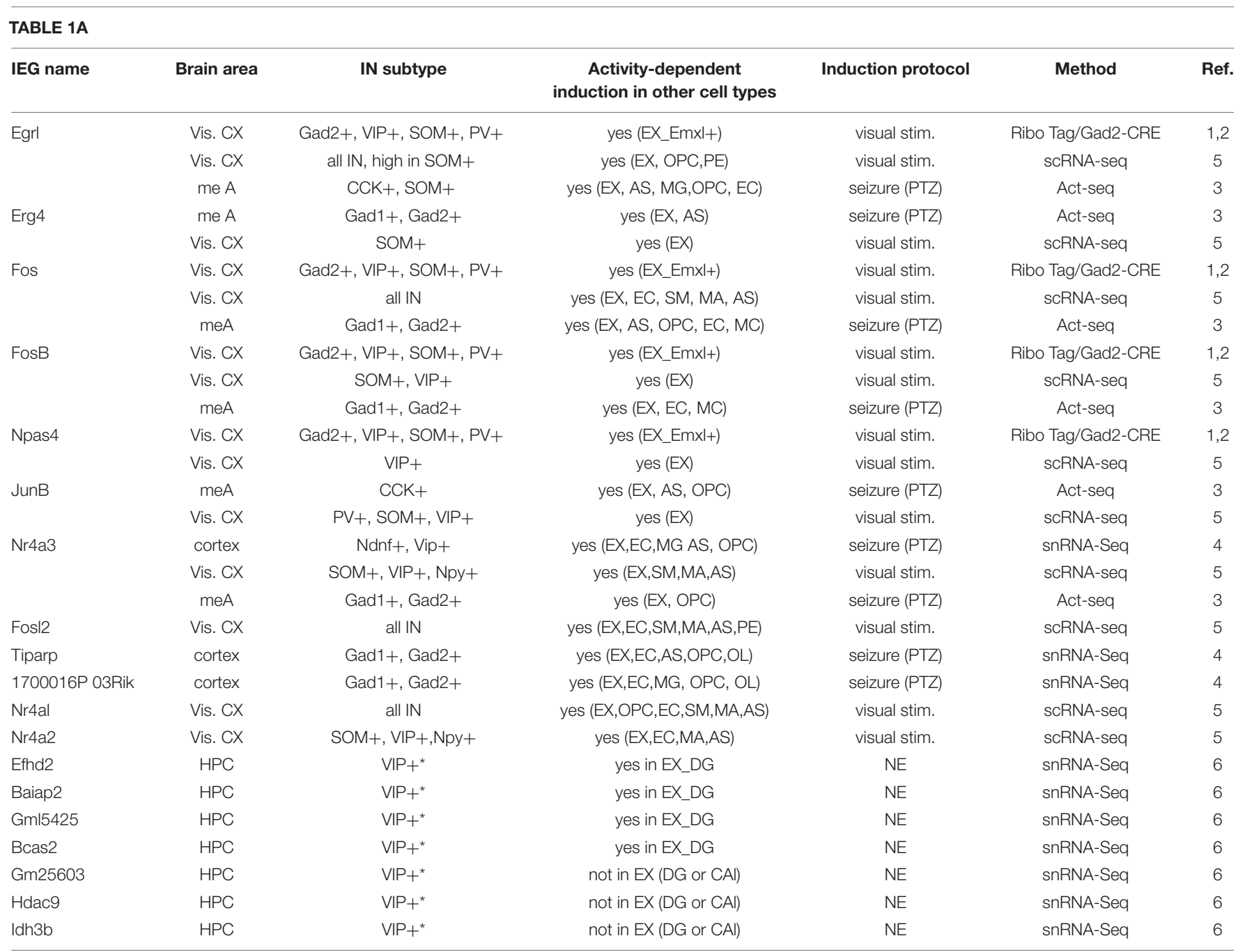

TABLE IB

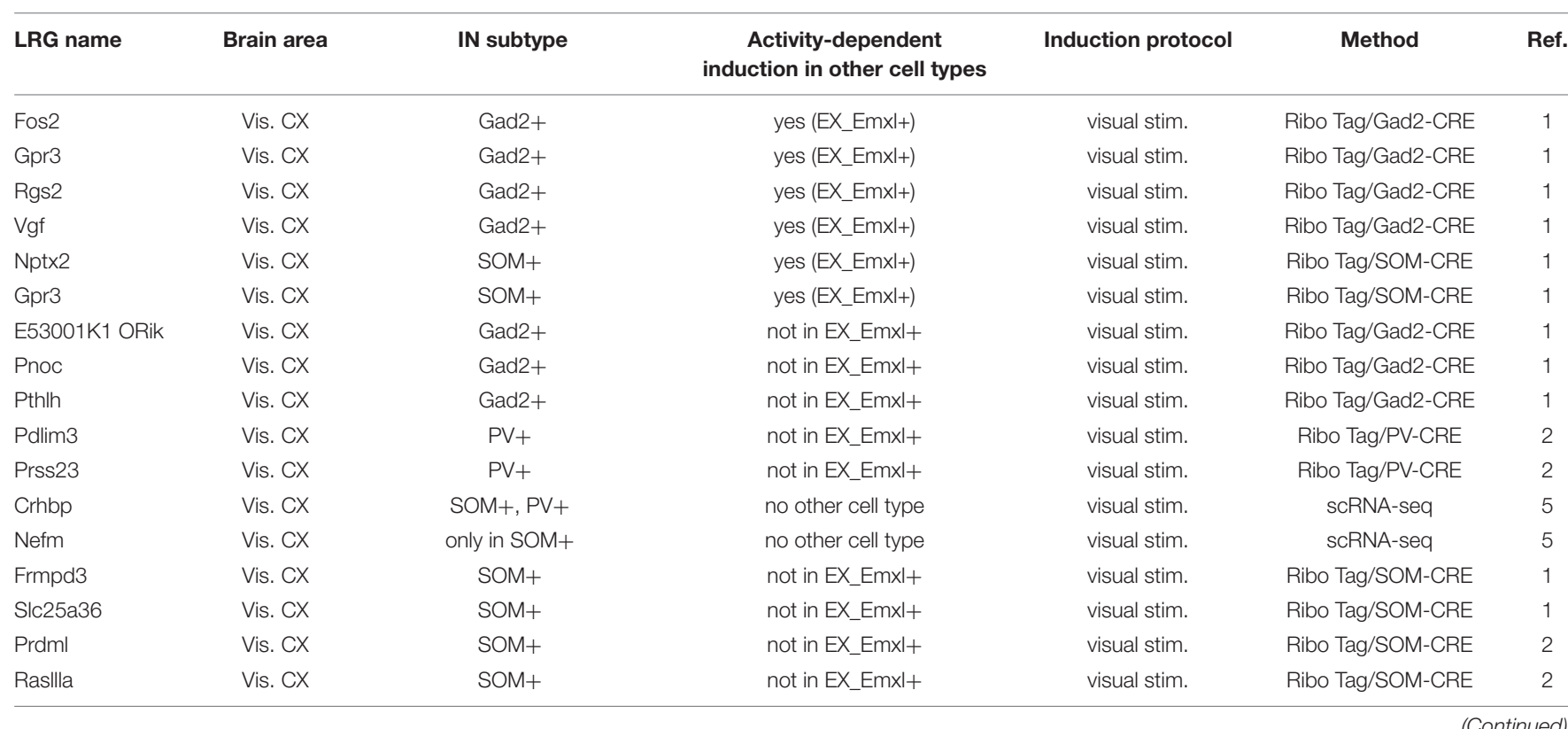


TABLE 1 | Continued

\begin{tabular}{|c|c|c|c|c|c|c|}
\hline \multicolumn{7}{|l|}{ TABLE 1B } \\
\hline LRG name & Brain area & IN subtype & $\begin{array}{l}\text { Activity-dependent } \\
\text { induction in other cell types }\end{array}$ & Induction protocol & Method & Ref. \\
\hline Serpinel & Vis. CX & SOM+ & not in EX_Emxl+ & visual stim. & Ribo Tag/SOM-CRE & 2 \\
\hline \multirow[t]{2}{*}{ Crh } & Vis. CX & $\mathrm{VIP}+, \mathrm{SOM}+, \mathrm{PV}+$ & not in EX_Emxl+ & visual stim. & Ribo Tag/VIP-CRE & 2 \\
\hline & Vis. CX & $\mathrm{VIP}+, \mathrm{SOM}+, \mathrm{CCK}+$ & no other cell type & visual stim. & scRNA-seq & 5 \\
\hline \multirow[t]{3}{*}{ lgfl } & Vis. CX & Gad2+ & not in EX_Emxl+ & visual stim. & Ribo Tag/Gad2-CRE & 1 \\
\hline & Vis. CX & VIP+ & not in EX_Emxl+ & visual stim. & Ribo Tag/VIP-CRE & 2 \\
\hline & Vis. CX & only in VIP+ & no other cell type & visual stim. & scRNA-seq & 5 \\
\hline lgfbp5 & Vis. CX & only in VIP+ & no other cell type & visual stim. & scRNA-seq & 5 \\
\hline Serpine2 & Vis. CX & only in VIP+ & no other cell type & visual stim. & scRNA-seq & 5 \\
\hline Gpdl & Vis. CX & VIP+ & not in EX_Emxl+ & visual stim. & Ribo Tag/VIP-CRE & 2 \\
\hline Hnflb & Vis. CX & $\mathrm{VIP}+$ & not in EX_Emxl+ & visual stim. & Ribo Tag/VIP-CRE & 2 \\
\hline Lpar2 & Vis. CX & $\mathrm{VIP}+$ & not in EX_Emxl+ & visual stim. & Ribo Tag/VIP-CRE & 2 \\
\hline Pde3a & Vis. CX & $\mathrm{VIP}+$ & not in EX_Emxl+ & visual stim. & Ribo Tag/VIP-CRE & 2 \\
\hline Prok2 & Vis. CX & $\mathrm{VIP}+$ & not in EX_Emxl+ & visual stim. & Ribo Tag/VIP-CRE & 2 \\
\hline Scgn & Vis. CX & $\mathrm{VIP}+$ & not in EX_Emxl+ & visual stim. & Ribo Tag/VIP-CRE & 2 \\
\hline Uts $2 r$ & Vis. CX & $\mathrm{VIP}+$ & not in EX_Emxl+ & visual stim. & Ribo Tag/VIP-CRE & 2 \\
\hline Aqp5 & Vis. CX & VIP+ & not in EX_Emxl+ & visual stim. & Ribo Tag/VIP-CRE & 2 \\
\hline
\end{tabular}

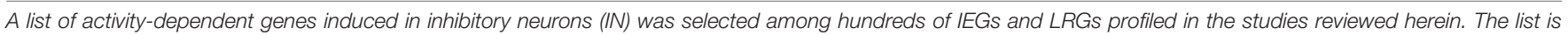

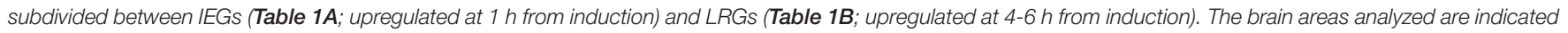

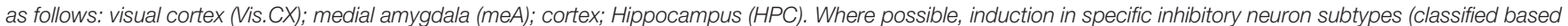

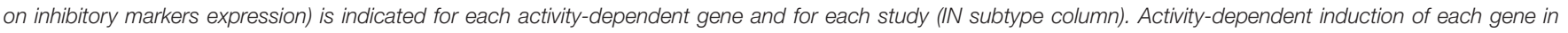

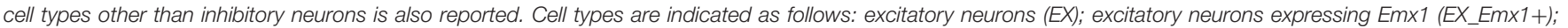

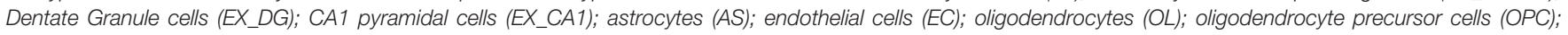

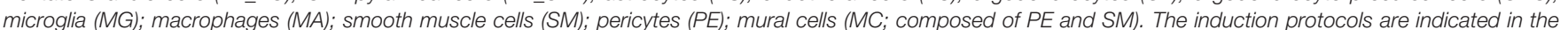

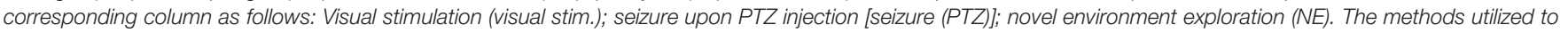

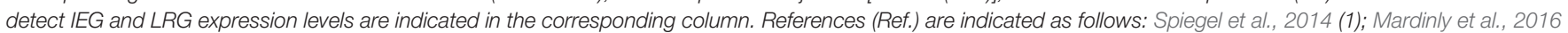

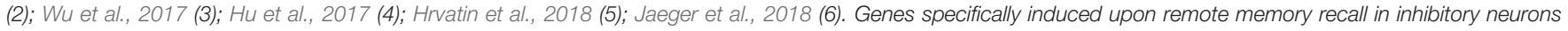
(Chen et al., 2020) are not reported. The asterisk indicates that VIP+ neurons were the only inhibitory subtype profiled in the study.

Coutellier et al., 2012; Bloodgood et al., 2013). Notably, the increase of inhibitory synapses onto the soma of pyramidal neurons elicited by Npas4 requires Bdnf, whose induction is restricted to excitatory neurons. Thus, Npas4 represents an ideal example of a shared IEG transcription factor that may induce distinct transcriptional responses in distinct neuronal subtypes. Depleting its expression in SOM+ interneurons specifically, the authors (Spiegel et al., 2014) find that Npas4 does not affect the number of inhibitory synapses formed onto these cells, in stark contrast with its role in excitatory neurons. To the contrary, Npas4 expression is required to promote formation of excitatory synapses onto $\mathrm{SOM}+$ neurons in response to activation, likely by eliciting expression of LRGs which participate in modifying excitatory synaptic inputs. Indeed, a characterization of Npas4regulated LRGs in excitatory and inhibitory neurons, confirms that this transcription factor induces distinct transcriptional programs in the two neuronal subtypes. In particular, four LRGs (Frmpd3, scl25a36, Kcna1, Ddhd1) were identified as specifically induced in SOM+ neurons activated in vivo, and several of these have been suggested to be involved in excitatory synapse formation and stabilization. Overall, these results suggest that in excitatory and inhibitory neurons activity triggers a common set of IEG which, in turn, elicit the expression of different combinations of LRGs resulting in subtype-specific synaptic responses to activity (Table 1). This mechanism is well exemplified by the role played by the IEG Npas4 in the homeostatic response to neuronal activation (Figure 1); while in excitatory neurons it elicits an increase in Bdnf expression and a recruitment of inhibitory inputs, in SOM+ GABAergic neurons it induces a different LRG transcriptional response resulting in an increase of excitatory inputs onto these cells. Interestingly, a follow-up study by the Greenberg laboratory revealed that, in the visual cortex, the transcriptional and functional response to activation of various interneuronal subclasses differ substantially (Mardinly et al., 2016). Using the Ribo-Tag approach, the authors profiled actively translated mRNAs at different time points from visual stimulation in $\mathrm{SOM}+\mathrm{VIP}+$, and $\mathrm{PV}+$ interneurons, yielding 31 genes that are both regulated by sensory experience and specific to a neuronal subtype. Remarkably, most of these cell-type specific activity-dependent genes encode proteins that are secreted and could thus play a role in modulating the synaptic connectivity of the activated interneuron. For example, of the 11 experience-regulated genes specific to VIP+ neurons, four code for secreted molecules and are Igf1, Crh, Prok2, and Fbln2. FISH analyses then revealed that Fbln2 is expressed at undetectable levels, while Prok2 is sparsely expressed only by a subset of VIP+ neurons upon stimulation of the visual cortex. Crh was instead induced in all VIP+ neurons, but also in other SOM+/PV+/VIP-negative interneurons. The authors thus proceed to investigate the function of Igf1, which 


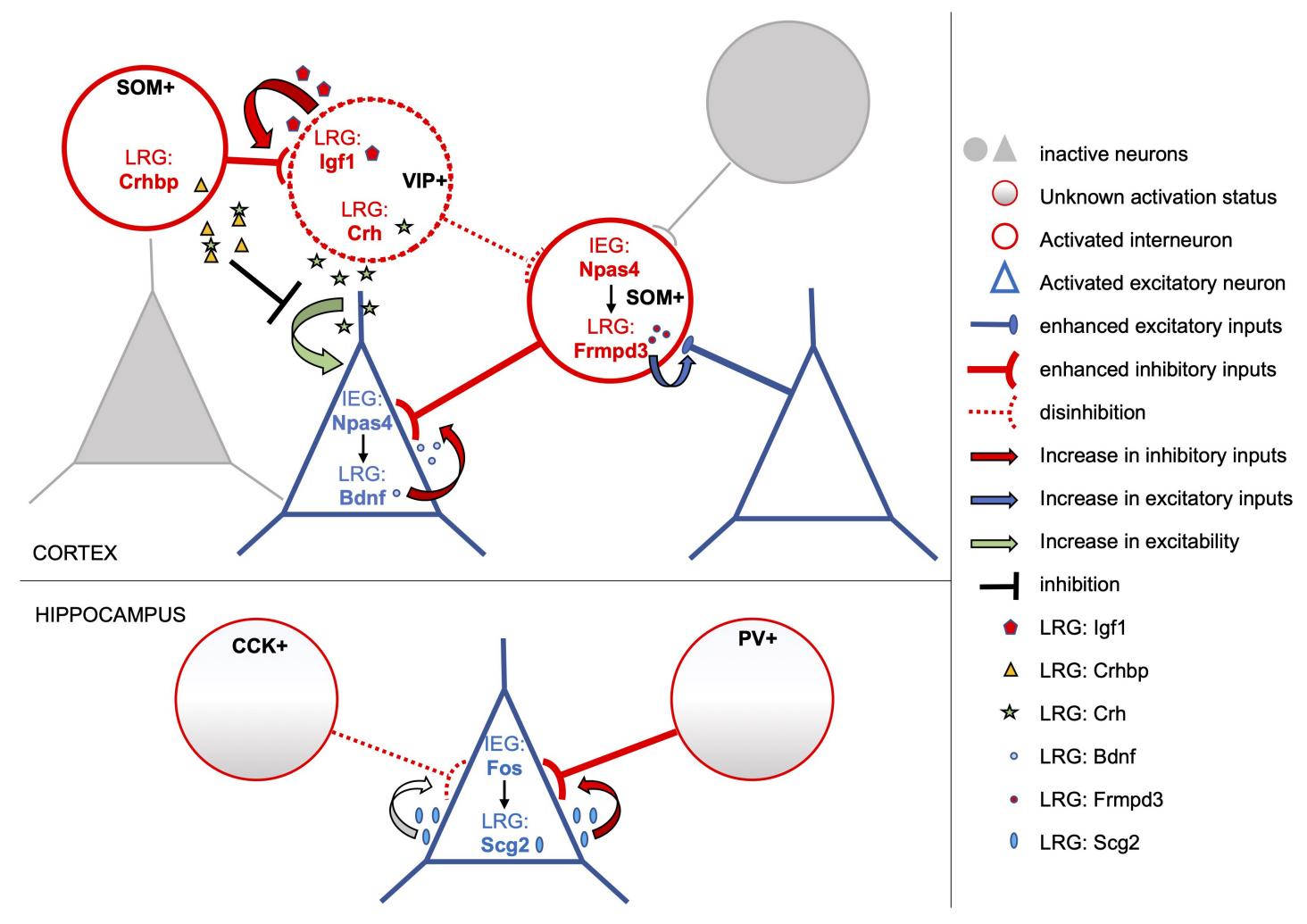

FIGURE 1 | Subtype-specific activity-dependent transcriptional programs shape circuit rearrangements in response to experience. The model depicts examples of activity-induced LRGs which modulate connectivity and/or excitability of distinct neuronal subtypes, overall enhancing the inhibitory tone onto activated pyramidal neurons. Excitatory and inhibitory neurons of the activated neuronal ensemble are depicted as blue triangles and red circles, respectively. In cortical excitatory neurons the IEG Npas4 drives expression of several LRGs including Bdnf (blue dots), which in turn promote an increase in the number of inhibitory inputs onto these cells. Conversely, Npas4 expression in activated SOM + inhibitory neurons triggers a transcriptional program that includes the LRG Frmpd3 (red dots), which induces an increase in excitation onto the GABAergic cell (Spiegel et al., 2014). Activity-dependent transcriptional profiles of cortical VIP + interneurons include the LRGs Igf1 (red pentagons) and Crh (green stars). Secreted lgf1 promotes inhibition of VIP + neurons (Mardinly et al., 2016), which often operate by disinhibiting principal neurons. On the other hand, VIP + neurons also release Crh which can increase the excitability of nearby pyramidal cells. However, this effect may be tampered by Crhbp (yellow triangles), an inhibitor of Chr secreted by SOM + neurons in response to stimulation (Hrvatin et al., 2018). Overall, activity-dependent transcriptional responses of both excitatory and inhibitory cortical neurons appear to converge in increasing the inhibitory tone onto activated excitatory neurons. Similarly, in the hippocampus, Fos expression in activated excitatory neurons induces the LRG Scg2, a precursor to four neuropeptides, which increases perisomatic inhibition by PV-interneurons albeit weakening that of CCK-interneurons (Yap et al., 2021).

is specifically enriched in most stimulated VIP+ neurons. Its selective depletion and overexpression in these cells revealed that Igf- 1 acts locally to promote the number and/or strength of inhibitory synapses formed onto VIP+ neurons in response to activity and does so in a cell autonomous manner (Figure 1). Further, Mardinly et al. (2016) show that experience-dependent induction of Igf-1 negatively regulates visual acuity. Since VIP+ neurons are involved in disinhibition (Pi et al., 2013) of cortical circuits, this study indicates that IGF-1 is secreted locally by VIP+ neurons to enhance their own inhibition, thereby putting a brake on the excitability of targeted principal neurons in response to experience.

\section{Activity-Dependent Transcription Studies With Single-Cell Resolution}

These pioneer studies radically advanced our understanding of activity-dependent transcriptional programs in inhibitory and excitatory neurons and started deciphering the molecular mechanisms that link experience-dependent transcriptional responses to the long-lasting synaptic adaptations of different neuronal subtypes. However, the availability of only a few subtype-specific genetic tools, such as the mouse CRE lines, limited the depth and breadth of these analyses, not capturing the full range of cell-types that compose the nervous system and that respond to activity. Only the advent of RNA sequencing from single-nuclei (snRNA-seq) or single-cells (scRNA Seq) could allow such a comprehensive analysis, and several recent studies have undertaken this important task with significant results. Pioneering this field, Lacar et al. (2016) investigated the transcriptional response of hippocampal dentate granule cells (DGC) at a single-neuron level in mice exposed to a novel environment. When first adopting common whole-cell dissociation protocols, the authors found that the conventional protease digestion step was alone able to induce IEG expression unspecifically and to a degree similar that observed upon 
seizure. To overcome this hurdle, they resorted to douncehomogenization of the tissue and FACS isolation of nuclei upon immunostaining for neuronal and DGC markers. Subsequent transcriptome profiling of these nuclei and sorting based on FOS expression, revealed that this approach allows for the detection of IEG expression in behaviorally activated DGC neurons, largely confirming the induction of known IEGs (Arc, Fos, and Erg1) and of their downstream targets. A similar approach was also utilized in a subsequent study by $\mathrm{Hu}$ et al. (2017) to resolve the transcriptional heterogeneity of cortical neuronal and non-neuronal cells. In this case, the authors combined the Drop-Seq microfluidic technique (Macosko et al., 2015) with single-nucleus RNA Seq. Also, nuclei from mouse cortices were sucrose-gradient purified rather than isolated by flow cytometry. When applying this novel "sNucDropSeq" method to interrogate the transcriptional response to Pentylenetetrazole (PTZ)-induced seizures, the authors find that among the inhibitory neuronal clusters identified, the two subtypes more likely to express activity-dependent genes are the $\mathrm{SOM}+$ and Ndnf + interneurons. Conversely, and in agreement with previous and subsequent reports (Sørensen et al., 2016; Hrvatin et al., 2018), PV+ interneurons are relatively less transcriptionally responsive to stimulation. Characterization of nuclear rather than whole-cell RNAs allows to better capture the dynamic and transitory expression profiles of activity-dependent genes, as it is not tainted by exported mRNAs which may endure for hours in the cytoplasm. This advantage provided for an additional important finding of this study regarding the timing of activity-dependent transcription in inhibitory neurons. In particular, $\mathrm{Hu}$ et al. observed that at $1 \mathrm{~h}$ from PTZ treatment the nuclei of inhibitory and excitatory neurons were differentially enriched of IEG and LRG transcripts, with GABAergic cells expressing IEGs (i.e., Fos and Erg1) while principal neurons were already engaged in transcribing LRGs such as Bdnf, $\mathrm{Mbnl} 2$, and Nptx2. The observed delay in the transcriptional response of inhibitory neurons suggests that their activation may be triggered subsequently to that of excitatory neurons, in accordance with a current model of inhibitory engram formation as a homeostatic response to the activation of excitatory memory engrams (Barron et al., 2017).

To obtain whole-cell transcriptomic profiles, $\mathrm{Wu}$ et al. resolved the issue of unspecific IEG expression induced by conventional dissociation methods with a different strategy (Wu et al., 2017). They observed that such spurious expression was minimized when the protease digestion step was performed at lower temperatures and in the presence of the transcriptional inhibitor actinomycin. They applied this protocol to analyze, at a single-cell level, the transcriptional profiles of cells composing the medial amygdala (MeA), in control animals and in animals undergoing either PTZ-induced seizures or behavioral stimuli like acute stress. Strikingly, they found that activity-induced IEG expression is not a prerogative of neurons and that partially overlapping sets of IEGs can be also detected in many nonneuronal cells, including astrocytes, microglia, oligodendrocytes, endothelial cells and mural cells. When focusing on neuronal subtypes, the authors found that a common set of four IEGs, Fos, Fosb, Egr4, and Nr4a3 (Table 1), was induced by seizure across all MeA neuronal subclusters, partially confirming previous data from Spiegel et al. (2014). However, most of the IEGs characterized displayed differential induction levels in the different clusters. Additionally, the authors were able to identify two neuronal subclusters, both expressing CCK, which were preferentially enriched of IEGs in response to acute stress. Shortly after, a similar study was published investigating the transcriptional response to sensory stimulation of the visual cortex at a single-cell level (Hrvatin et al., 2018). As in the MeA, also in the visual cortex a large array of non-neuronal cells displays strong transcriptional responses to stimulation, particularly noticeable in endothelial and smooth muscle cells, astrocytes, pericytes and macrophages. Further, the authors (Hrvatin et al., 2018) identify 611 genes significantly regulated by sensory stimulation in the various cell types, divided into 362 early-response and 249 late-response genes. When examining their expression levels across the 30 different subtype clusters, they observed great heterogeneity in their distribution, highlighting divergent transcriptional responses both at early and late time points from light stimulation. Interestingly, among 38 IEG transcription factors identified, only half are expressed by three or more cell types, meaning that a large portion of IEGs is expressed only in defined clusters. Of the "shared" IEG transcription factor, a small subset (including Fos, Fosl2, Erg1, Nr4a1-3) are induced in both neuronal and non-neuronal cell types, while five (Npas4, Junb, Fosb, Erg2 and Erg4) are induced only in neurons, particularly in all excitatory sub-clusters and in the SOM+ and VIP+ inhibitory sub-clusters (Table 1). Hence, a more in depth single-cell analysis of experience-regulated gene expression profiles depicts a picture that partially diverges from the initial conclusions drawn by Spiegel et al. (2014). Indeed, if on one hand the existence of a small core of common early transcription factors is confirmed, on the other, the bulk of early transcriptional responses appears to be highly diversified across cell-types and could contribute to drive differential downstream LRG programs. When examining sensory-induced LRG programs, both excitatory and inhibitory sub-clusters display highly divergent transcriptional profiles. In particular, the authors (Hrvatin et al., 2018) were able to identify 14 late-response genes differentially enriched in specific inhibitory subtypes; for example, the LRGs Igf1, Crh, Igfbp5 and Serpin2 are specifically enriched in VIP+ neurons (Table 1). While the role of Igf1 had been previously examined (Mardinly et al., 2016), in this study the authors point to Crh (the Corticotropin-releasing hormone) as a molecule secreted by VIP+ neurons which can increase the excitability of nearby pyramidal cells in response to stimulation (Figure 1). Conversely, SOM+ interneurons secrete an inhibitor of Crh, suggesting a novel mechanism by which sensory-experience shapes neuronal excitability through the opposing effects of LRG-encoded molecules secreted by two different inhibitory subtypes (Figure 1).

Alternative approaches to scRNA-seq, that resolve not only the identity but also the spatial distribution of activity-dependent transcripts, are also emerging (Lein et al., 2017). These include the 3D intact-tissue RNA sequencing "STARmap" methodology, developed by combining hydrogel-tissue chemistry, targeted signal amplification and in situ sequencing (Wang et al., 2018). 
With this approach, Wang et al. (2018) were able to obtain a 3D map single-cell transcriptomic profiles and spatial organization of molecularly defined cell types. This promising technique was also applied to assess the expression pattern of 48 activity dependent genes in the visual cortex at $1 \mathrm{~h}$ of visual stimulation. The results were highly consistent with previous scRNA-Seq data, reaffirming the existence of a small set of common IEGs (including Fos and Egr1) and a significant divergence in the expression of all other IEG tested across cell-types. Further, the authors could observe a more pronounced diversification of IEG transcriptional profiles across inhibitory neuron subtypes than across excitatory neuron subtypes, again confirming the conclusions drawn by scRNA-Seq studies.

Finally, a recently published study investigated how activitydependent transcription, triggered by exploration of a novel environment, drives circuit reorganization by modulating perisomatic inhibitory plasticity onto CA1 pyramidal hippocampal neurons (Yap et al., 2021). The authors focus on the Fos-dependent transcriptional cascade in these cells to characterize LRGs that are responsible for the observed increase in inhibition by $\mathrm{PV}$-interneurons and weakening of CCK-interneurons inputs. To this end, they combine the results of three different profiling approaches; a Ribo-tag analysis of activity-dependent genes in CamkII-positive cells, a snRNA-seq of Fos-depleted nuclei upon kainic acid stimulation and finally, a chromatin-profiling strategy to identify Fos-bound target genes. Six candidate effector LRGs, induced by Fos in CA1 excitatory neurons, stand out from these analyses: Inhba, Bdnf, Scg2, Rgs2, Nptx2 and Pcsk1. Of these, the pro-neuropeptide Scg2 emerged as the Fos-dependent LRG key to modulating perisomatic inhibition by PV and CCK interneurons and able to affect network rhythms. Thus, in CA1 pyramidal neurons, activitydependent expression of Fos leads to selective induction of Scg2, a precursor to four neuropeptides, which in turn rearranges PV and CCK inhibitory inputs (Figure 1), likely facilitating memory consolidation. This study also revealed that, as observed for Npas4 (Spiegel et al., 2014), Fos-dependent LRG programs differ substantially in the various cell-types analyzed. Consequently, it would be interesting to learn whether Fos-dependent LRGs specifically induced in inhibitory neurons intervene to modulate synaptic plasticity, and possibly network oscillations, similarly to what observed in pyramidal neurons via the LRG Scg2.

\section{Transcriptional Programs in Memory Recall and Consolidation}

The studies presented above all focused on characterizing the transcriptional programs of activated cells within the first few hours from the inducing stimuli, which ranged from pharmacological induction to visual stimulation and novel environment exploration (Table 1). The transcriptional programs characterized are therefore potentially useful to better understand, and possibly manipulate, the acquisition phase of memory encoding or learning. Hence, the question arises as to what happens in the subsequent phases of memory formation and recall. Is activity-dependent transcription modulated during the process of engram selection, consolidation and reactivation, and what is its contribution to each specific stage? Two recent studies started addressing these questions. Adopting the snRNA-seq technique (Lacar et al., 2016; Jaeger et al., 2018) characterized the transcriptional profile of specific subsets of Fos + hippocampal neurons (CA1 pyramidal neurons, VIP+ interneurons and DGC) at early and late ( 1 and $5 \mathrm{~h}$ ) time points from novel environment exposure and upon re-exposition to the same, or a different, environment (after $4 \mathrm{~h}$ from the initial exposure). Among several important findings of this study, the authors were able to define an early activity-induced transcriptional signature of DGC neurons that is predictive of their reactivation in the recall phase of the behavioral paradigm; what could be seen as a transcriptional mark for engram cell selection. Further, they find that a significant portion of the transcriptional response of activated DG neurons is devoted to reducing excitability and increasing tonic inhibition of these cells. This evidence suggests an intriguing mechanism of engram ensemble selection in the DG. Activation of the initial network of DG neurons triggers heterogeneous transcriptional programs which also dampen excitability of the activated neurons: only those neurons expressing specific gene patterns can overcome such heightened inhibition and are selected as engram cells encoding that memory. With regard to inhibitory neurons, the authors report that hippocampal Fos + VIP+ interneurons are transcriptionally less responsive to novel environment exposure compared to CA1 glutamatergic neurons and to DGC. Only seven experience-dependent IEGs appear induced in Fos+ VIP+ neurons (Table 1A), while ten are downregulated (Jaeger et al., 2018). This result diverges from what observed in the visual cortex upon sensory stimulation (Mardinly et al., 2016; Hrvatin et al., 2018) possibly reflecting differences in the transcriptional responsiveness of cortical versus hippocampal VIP interneurons. Alternatively, selection of activated neurons based on Fos expression may exclude neurons which do not express Fos but are nonetheless transcriptionally active (Sun et al., 2020). Unfortunately, additional studies that provide a comprehensive single-cell analysis of the transcriptional responses in inhibitory neurons upon memory formation are lacking. Conversely, studies that specifically address memory-induced transcriptional programs in activated excitatory neurons are starting to emerge (Rao-Ruiz et al., 2019; Yap et al., 2021).

However, a recent study was published which directly investigated the transcriptional profiles of cortical engram cells activated 16 days post-training, in a fear memory recall paradigm (Chen et al., 2020). This first profiling of consolidated engram cell composition, and of gene expression patterns associated with remote memory recall, brought important findings to light. First, that the relative composition in neuronal subtypes of active and inactive neurons is highly similar, albeit for an enhanced recruitment of Calb2+ GABAergic interneurons within the reactivated ensemble. Secondly, that cell type-specific persistent transcriptional programs are elicited during memory consolidation in both neuronal and nonneuronal engram cells, such as astrocytes and microglia. These gene expression profiles are long lasting and likely contribute to maintaining the memory trace at remote time 
points after learning. Intriguingly, the authors also find that within these memory-related transcriptional programs, a large portion of activated genes are related to vesicle-mediated transport, exocytosis and neurotransmitter secretion. This implies a strong participation of these cellular processes in memory consolidation.

\section{CONCLUSION AND PERSPECTIVES}

Overall, in recent years high-throughput approaches have significantly accelerated the discovery of stimulus-dependent transcriptional profiles across a surprisingly vast array of neuronal and non-neuronal cell-types. It needs to be noted that, with a few recent exceptions, most of these investigations interrogated the transcriptional response of heterogenous brain areas to pharmacological or sensory stimulation, not to memoryinducing protocols. This is particularly true with respect to GABAergic neurons (Table 1). To our knowledge, only one single-cell transcriptomic study profiled the transcriptional program of inhibitory neurons upon memory formation (Jaeger et al., 2018), limiting the analysis to the hippocampal Fos + VIP + cluster. Indeed, the study by Chen et al. (2020) specifically investigated the transcriptional signature of both inhibitory and excitatory neurons upon remote memory recall. Moreover, the lack of uniformity in experimental approaches, while enriching, complicates an already intricate picture of sub-type specific transcriptional responses to activity. Hence, it is premature to ascribe specific transcriptional responses to different neuronal subclasses upon memory formation. Nonetheless, important hints can be extrapolated from these pioneer studies, albeit requiring further validation in memory-related processes. Except for a small set of immediate-early genes expressed in multiple cell-types, both the early and late transcriptional responses appear to be markedly subtype-specific. This feature is particularly noticeable across the different classes of interneurons, likely reflecting a higher degree of functional diversification among GABAergic subtypes. Further, this diversity will surely be instrumental in the development of genetic tools to map and manipulate the activation of specific subtypes of neuronal and nonneuronal cells.

The significance of these studies, however, goes well beyond the potential advancement in memory trace labeling techniques. As described above, characterization of cell typespecific transcriptional responses to activity has led to the unveiling of complex and varied molecular cascades that underlie stimulation-dependent circuit reorganization. This is best exemplified by the opposite effects on synaptic connectivity triggered by Npas4 in excitatory versus inhibitory neurons (Figure 1). Similarly, Fos appears to induce highly diversified LRG transcriptional programs specific to each cellular subtype, likely mediating different rearrangements of their synaptic connections within their neuronal network. Additional complexity stems from heterogeneous IEG expression, as suggested by the identification of distinct engram ensembles in the dentate gyrus whose function and circuit reorganization are differentially directed by Fos and Npas4 transcriptional programs (Sun et al., 2020).

A common theme that emerges from these investigations, is that activity-dependent transcriptional programs are elicited and persist in both neuronal and non-neuronal cells, particularly in astrocytes and microglia. Thus, memory engrams likely consist of a heterogeneous network of cells, including excitatory neurons, inhibitory neurons and, intriguingly, glia cells. In support of this, several functional studies point to microglia, astrocytes and oligodendrocytes, as fundamental cellular subtypes driving and sustaining plasticity and memory (Parkhurst et al., 2013; Adamsky et al., 2018; Nguyen et al., 2020; Pan et al., 2020; Steadman et al., 2020; Zhou et al., 2021).

Another aspect to which several studies converge, is that activity-dependent transcriptional responses, in both excitatory and inhibitory neurons, are often directed at increasing the number or strength of inhibitory inputs onto activated pyramidal neurons (Bloodgood et al., 2013; Spiegel et al., 2014; Mardinly et al., 2016; Hrvatin et al., 2018; Jaeger et al., 2018; Sun et al., 2020; Yap et al., 2021). Consequently, the question arises: how does an increased inhibitory drive onto the activated excitatory ensemble contribute to engram formation? A possible answer is that this homeostatic response may represent a mechanism for the allocation of the inhibitory component of the engram and for shaping engram size, whereby inhibitory engram neurons are recruited by, and in response to, activation of excitatory ensemble neurons. This hypothesis would be also in accordance with a model of "inhibitory engram" formation proposed by Barron et al. (2017) and with the observation of a delayed transcriptional response to activity in inhibitory neurons (Hu et al., 2017).

Nonetheless, an increased inhibitory tone onto activated pyramidal cells needs to be reconciled with consolidated evidence showing that heightened intrinsic excitability of activated ensemble neurons is critical for their allocation to the engram (reviewed in Yiu et al., 2014; Josselyn and Frankland, 2018; Josselyn and Tonegawa, 2020). Thus, how can heightened excitability and increased inhibitory tone coexist in activated ensemble pyramidal neurons? And how do they functionally cooperate to the process of engram allocation? As suggested above (Morrison et al., 2016; Stefanelli et al., 2016; Jaeger et al., 2018), heightened inhibition of activated glutamatergic neurons may contribute to restrict engram allocation to a discrete subset of activated neurons, likely those with an increased intrinsic excitability. In this light, we propose two possible solutions to this apparent paradox.

In a first scenario (Figure 2, hypothesis A), an increase in inhibitory inputs could be a generalized response to the initial stimuli, targeting pyramidal neurons of the activated ensemble. Only those neurons with a heightened intrinsic excitability would be able to override this increased inhibitory drive and win the competition for engram allocation. In this scenario, those inhibitory neurons targeting successfully allocated engram cells could become the inhibitory component of that engram, controlling its storage, latency and recall.

In an alternative hypothesis (Figure 2, hypothesis B), excitability and inhibition may act on distinct subsets of activated pyramidal neurons to restrict engram size. Those 


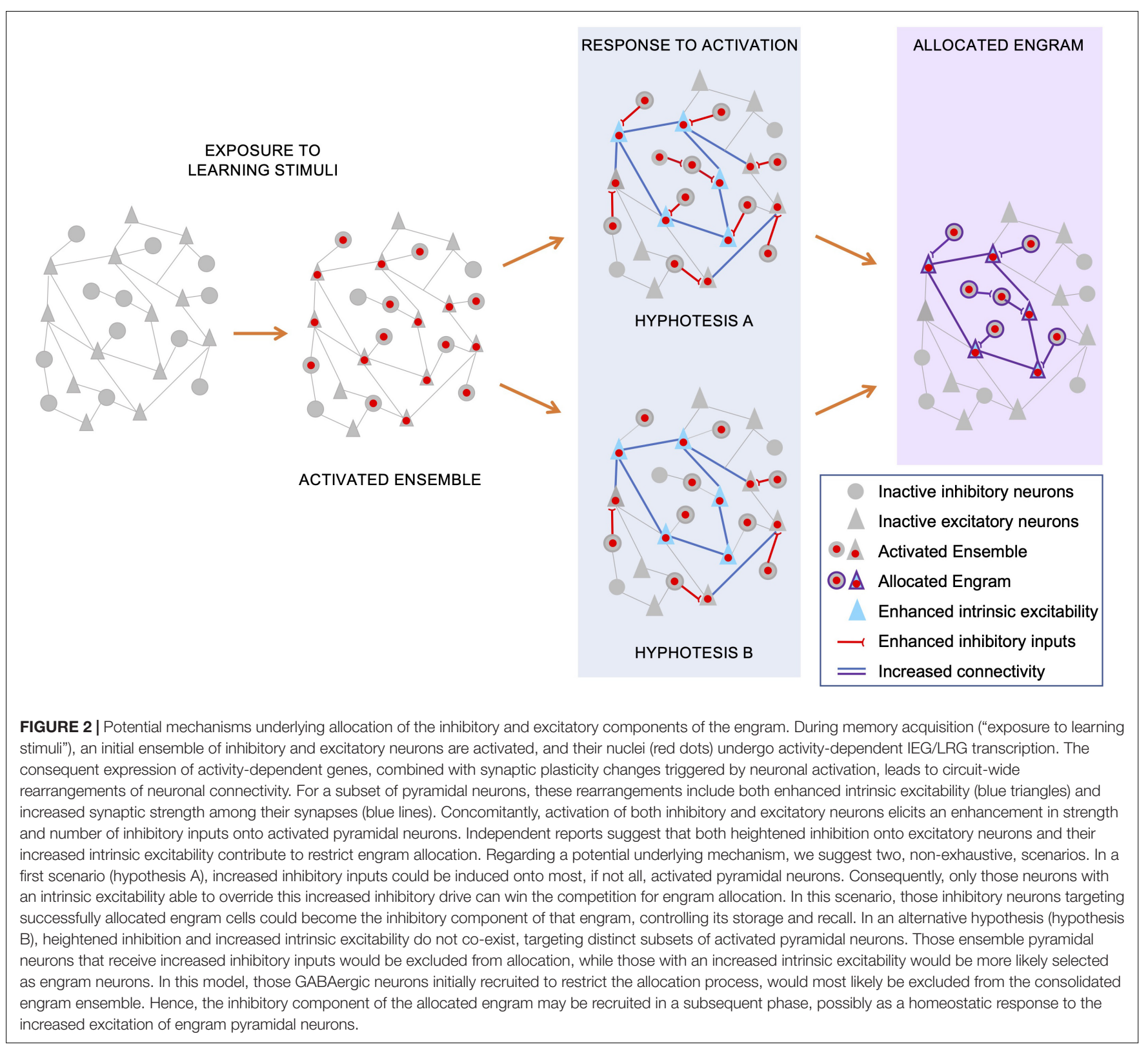

ensemble pyramidal neurons that receive increased inhibitory inputs would be largely excluded from allocation, while those with an increased intrinsic excitability would be more likely selected as engram neurons. In this scenario, those inhibitory neurons initially recruited to restrict the allocation process, would most likely be excluded from the consolidated engram ensemble. In this case, the inhibitory component of the allocated engram may be recruited in a homeostatic response to the increased excitation of engram pyramidal neurons.

These proposed allocation mechanisms are clearly nonexhaustive. Nonetheless, to test them it would be useful to ascertain whether pyramidal neurons that are activated during memory acquisition, and that display increased intrinsic excitability, also receive increased inhibitory inputs.
Numerous other aspects regarding the recruitment and activity of the inhibitory component of memory engrams remain unclear. For instance, it would be useful to determine if those inhibitory neurons that are activated in the first phase of engram allocation are the same inhibitory cells that control storage and recall of that memory trace. Further investigations are also needed to establish the role played by disynaptic disinhibition in these processes. It is conceivable that disinhibitory mechanisms may play a key role in memory recall, relieving inhibition and allowing reactivation of excitatory engram cells. Similarly, disinhibition may contribute to engram allocation by enhancing excitation of pyramidal engram cells. However, compelling experimental evidence that support these hypotheses is still lacking. The rapid expansion of studies on the role of inhibitory neurons in memory processes, 
and the constant development of new genetic tools that can access engram cells with subtype specificity, will undoubtedly provide clues to address these challenging questions. Finally, transcriptomic analyses have unveiled experience-dependent widespread activation of non-neuronal cells, including glia, endothelial cells and macrophages. Understanding how the activity of these cells may contribute to modulate both excitatory and inhibitory engram neurons is surely a new and intriguing area to investigate in memory engram studies.

\section{AUTHOR CONTRIBUTIONS}

Both authors conceived and wrote the manuscript and approved the submitted version.

\section{REFERENCES}

Abraham, W. C., Jones, O. D., and Glanzman, D. L. (2019). Is plasticity of synapses the mechanism of long-term memory storage. NPJ Sci. Learn. 4:9.

Adamsky, A., Kol, A., Kreisel, T., Doron, A., Ozeri-Engelhard, N., Melcer, T., et al. (2018). Astrocytic Activation Generates De Novo Neuronal Potentiation and Memory Enhancement. Cell 174, 59-71.e14. doi: 10.1016/j.cell.2018.05.002

Alberini, C. M., and Kandel, E. R. (2014). The regulation of transcription in memory consolidation. Cold Spring Harb. Perspect. Biol. 7:a021741. doi: 10. 1101/cshperspect.a021741

Artinian, J., and Lacaille, J. C. (2018). Disinhibition in learning and memory circuits: New vistas for somatostatin interneurons and long-term synaptic plasticity. Brain Res. Bull. 141, 20-26. doi: 10.1016/j.brainresbull.2017.11.012

Barron, H. C., Vogels, T. P., Behrens, T. E., and Ramaswami, M. (2017). Inhibitory engrams in perception and memory. Proc. Natl. Acad. Sci. U. S. A. 114, 6666-6674.

Barron, H. C., Vogels, T. P., Emir, U. E., Makin, T. R., O’Shea, J., Clare, S., et al. (2016). Unmasking Latent Inhibitory Connections in Human Cortex to Reveal Dormant Cortical Memories. Neuron 90, 191-203. doi: 10.1016/j.neuron.2016. 02.031

Bekinschtein, P., Cammarota, M., Igaz, L. M., Bevilaqua, L. R., Izquierdo, I., and Medina, J. H. (2007). Persistence of long-term memory storage requires a late protein synthesis- and BDNF- dependent phase in the hippocampus. Neuron 53, 261-277. doi: 10.1016/j.neuron.2006.11.025

Bloodgood, B. L., Sharma, N., Browne, H. A., Trepman, A. Z., and Greenberg, M. E. (2013). The activity-dependent transcription factor NPAS4 regulates domain-specific inhibition. Nature 503, 121-125. doi: 10.1038/nature 12743

Bontempi, B., Laurent-Demir, C., Destrade, C., and Jaffard, R. (1999). Timedependent reorganization of brain circuitry underlying long-term memory storage. Nature 400, 671-675. doi: 10.1038/23270

Chen, M. B., Jiang, X., Quake, S. R., and Südhof, T. C. (2020). Persistent transcriptional programmes are associated with remote memory. Nature 587, 437-442. doi: 10.1038/s41586-020-2905-5

Chiu, C. Q., Barberis, A., and Higley, M. J. (2019). Preserving the balance: diverse forms of long-term GABAergic synaptic plasticity. Nat. Rev. Neurosci. 20, 272-281. doi: 10.1038/s41583-019-0141-5

Chiu, C. Q., Martenson, J. S., Yamazaki, M., Natsume, R., Sakimura, K., Tomita, S., et al. (2018). Input-Specific NMDAR-Dependent Potentiation of Dendritic GABAergic Inhibition. Neuron 97, 368-377.e3.

Choi, J. H., Sim, S. E., Kim, J. I., Choi, D. I., Oh, J., Ye, S., et al. (2018). Interregional synaptic maps among engram cells underlie memory formation. Science 360, 430-435. doi: 10.1126/science.aas 9204

Clayton, D. F., Anreiter, I., Aristizabal, M., Frankland, P. W., Binder, E. B., and Citri, A. (2020). The role of the genome in experience-dependent plasticity: Extending the analogy of the genomic action potential. Proc. Natl. Acad. Sci. U. S. A. 117, 23252-23260. doi: 10.1073/pnas. 1820837116

\section{FUNDING}

This work was supported (in part) by the Fondo Ordinario Enti (FOE D.M 865/2019) funds in the framework of a collaboration agreement between the Italian National Research Council and EBRI (2019-2021) and Italian Ministry of Education, University and Research, PRIN project, prot. 2017HPTFFC.

\section{ACKNOWLEDGMENTS}

The authors are grateful to Cristina Marchetti for critical reading of the manuscript and Marilena Griguoli and Ivan Arisi for their helpful discussion.

Courtin, J., Chaudun, F., Rozeske, R. R., Karalis, N., Gonzalez-Campo, C., Wurtz, H., et al. (2014). Prefrontal parvalbumin interneurons shape neuronal activity to drive fear expression. Nature 505, 92-96. doi: 10.1038/nature12755

Coutellier, L., Beraki, S., Ardestani, P. M., Saw, N. L., and Shamloo, M. (2012). Npas4: a neuronal transcription factor with a key role in social and cognitive functions relevant to developmental disorders. PLoS One. 7:e46604. doi: 10. 1371/journal.pone.0046604

Cummings, K. A., and Clem, R. L. (2020). Prefrontal somatostatin interneurons encode fear memory. Nat. Neurosci. 23, 61-74. doi: 10.1038/s41593-0190552-7

Davenport, C. M., Rajappa, R., Katchan, L., Taylor, C. R., Tsai, M. C., Smith, C. M., et al. (2021). Relocation of an Extrasynaptic $\mathrm{GABA}_{A}$ Receptor to Inhibitory Synapses Freezes Excitatory Synaptic Strength and Preserves Memory. Neuron 109, 123-134. doi: 10.1016/j.neuron.2020.09.037

DeNardo, L., and Luo, L. (2017). Genetic strategies to access activated neurons. Curr. Opin. Neurobiol. 45, 121-129. doi: 10.1016/j.conb.2017.05.014

Disterhoft, J. F., Coulter, D. A., and Alkon, D. L. (1986). Conditioning-specific membrane changes of rabbit hippocampal neurons measured in vitro. Proc. Natl. Acad. Sci. U. S. A. 83, 2733-2737. doi: 10.1073/pnas.83.8.2733

Donato, F., Rompani, S. B., and Caroni, P. (2013). Parvalbumin-expressing basketcell network plasticity induced by experience regulates adult learning. Nature 504, 272-276. doi: 10.1038/nature12866

Duke, C. G., Kennedy, A. J., Gavin, C. F., Day, J. J., and Sweatt, J. D. (2017). Experience-dependent epigenomic reorganization in the hippocampus. Learn. Mem. 24, 278-288. doi: 10.1101/lm.045112.117

Fernandes, D., and Carvalho, A. L. (2016). Mechanisms of homeostatic plasticity in the excitatory synapse. J. Neurochem. 139, 973-996. doi: 10.1111/jnc.13687

Fernandez-Albert, J., Lipinski, M., Lopez-Cascales, M. T., Rowley, M. J., MartinGonzalez, A. M., Del Blanco, B., et al. (2019). Immediate and deferred epigenomic signatures of in vivo neuronal activation in mouse hippocampus. Nat. Neurosci. 22, 1718-1730. doi: 10.1038/s41593-019-0476-2

Frankland, P. W., Bontempi, B., Talton, L. E., Kaczmarek, L., and Silva, A. J. (2004). The involvement of the anterior cingulate cortex in remote contextual fear memory. Science 304, 881-883. doi: 10.1126/science.1094804

Frey, U., Frey, S., Schollmeier, F., and Krug, M. (1996). Influence of actinomycin D, a RNA synthesis inhibitor, on long-term potentiation in rat hippocampal neurons in vivo and in vitro. J. Physiol. 490, 703-711. doi: 10.1113/jphysiol. 1996.sp021179

Garner, A. R., Rowland, D. C., Hwang, S. Y., Baumgaertel, K., Roth, B. L., Kentros, C., et al. (2012). Generation of a synthetic memory trace. Science 335, 1513-1516. doi: 10.1126/science.1214985

Ghosh, S., Reuveni, I., Lamprecht, R., and Barkai, E. (2015). Persistent CaMKII activation mediates learning-induced long-lasting enhancement of synaptic inhibition. J. Neurosci. 35, 128-139. doi: 10.1523/jneurosci.2123-14.2015

Gobbo, F., Marchetti, L., Jacob, A., Pinto, B., Binini, N., Pecoraro Bisogni, F., et al. (2017). Activity-dependent expression of Channelrhodopsin at neuronal synapses. Nat. Commun. 8:1629. 
Goel, P., and Dickman, D. (2021). Synaptic homeostats: latent plasticity revealed at the Drosophila neuromuscular junction. Cell Mol. Life Sci. 8, 3159-3179. doi: 10.1007/s00018-020-03732-3

Goelet, P., Castellucci, V. F., Schacher, S., and Kandel, E. R. (1986). The long and the short of long-term memory-a molecular framework. Nature 322, 419-422. doi: $10.1038 / 322419 \mathrm{a} 0$

Gouty-Colomer, L. A., Hosseini, B., Marcelo, I. M., Schreiber, J., Slump, D. E., Yamaguchi, S., et al. (2016). Arc expression identifies the lateral amygdala fear memory trace. Mol. Psychiatry 21, 364-375. doi: 10.1038/mp.2015.18

Guenthner, C. J., Miyamichi, K., Yang, H. H., Heller, H. C., and Luo, L. (2013). Permanent genetic access to transiently active neurons via TRAP: targeted recombination in active populations. Neuron 78, 773-784. doi: 10.1016/j. neuron.2013.03.025

Guo, N., Soden, M. E., Herber, C., Kim, M. T., Besnard, A., Lin, P., et al. (2018). Dentate granule cell recruitment of feedforward inhibition governs engram maintenance and remote memory generalization. Nat. Med. 24, 438-449. doi: 10.1038/nm.4491

Halder, R., Hennion, M., Vidal, R. O., Shomroni, O., Rahman, R. U., Rajput, A., et al. (2016). DNA methylation changes in plasticity genes accompany the formation and maintenance of memory. Nat. Neurosci. 19, 102-110. doi: $10.1038 / \mathrm{nn} .4194$

Han, J. H., Kushner, S. A., Yiu, A. P., Cole, C. J., Matynia, A., Brown, R. A., et al. (2007). Neuronal competition and selection during memory formation. Science 316, 457-460. doi: 10.1126/science.1139438

Han, J. H., Kushner, S. A., Yiu, A. P., Hsiang, H. L., Buch, T., Waisman, A., et al. (2009). Selective erasure of a fear memory. Science 323, 1492-1496.

Hartzell, A. L., Martyniuk, K. M., Brigidi, G. S., Heinz, D. A., Djaja, N. A., Payne, A., et al. (2018). NPAS4 recruits CCK basket cell synapses and enhances cannabinoid-sensitive inhibition in the mouse hippocampus. Elife 7:e35927.

Hayashi-Takagi, A., Yagishita, S., Nakamura, M., Shirai, F., Wu, Y. I., Loshbaugh, A. L., et al. (2015). Labelling and optical erasure of synaptic memory traces in the motor cortex. Nature 525, 333-338. doi: 10.1038/nature15257

Holtmaat, A., and Caroni, P. (2016). Functional and structural underpinnings of neuronal assembly formation in learning. Nat. Neurosci. 19, 1553-1562. doi: $10.1038 / \mathrm{nn} .4418$

Hrvatin, S., Hochbaum, D. R., Nagy, M. A., Cicconet, M., Robertson, K., Cheadle, L., et al. (2018). Single-cell analysis of experience-dependent transcriptomic states in the mouse visual cortex. Nat. Neurosci. 21, 120-129. doi: 10.1038/ s41593-017-0029-5

Hu, P., Fabyanic, E., Kwon, D. Y., Tang, S., Zhou, Z., and Wu, H. (2017). Dissecting Cell-Type Composition and Activity-Dependent Transcriptional State in Mammalian Brains by Massively Parallel Single-Nucleus RNA-Seq. Mol. Cell. 68, 1006-1015.e7. doi: 10.1016/j.molcel.2017.11.017

Jaeger, B. N., Linker, S. B., Parylak, S. L., Barron, J. J., Gallina, I. S., Saavedra, C. D., et al. (2018). A novel environment-evoked transcriptional signature predicts reactivity in single dentate granule neurons. Nat. Commun. 9:3084.

Josselyn, S. A., and Frankland, P. W. (2018). Memory Allocation: Mechanisms and Function. Annu. Rev. Neurosci. 41, 389-413. doi: 10.1146/annurev-neuro080317-061956

Josselyn, S. A., Köhler, S., and Frankland, P. W. (2015). Finding the engram. Nat. Rev. Neurosci. 16, 521-534. doi: 10.1038/nrn4000

Josselyn, S. A., and Tonegawa, S. (2020). Memory engrams: Recalling the past and imagining the future. Science 367:eaaw4325. doi: 10.1126/science.aaw4325

Kandel, E. R. (2001). The molecular biology of memory storage: a dialogue between genes and synapses. Science 294, 1030-1038. doi: 10.1126/science.1067020

Karunakaran, S., Chowdhury, A., Donato, F., Quairiaux, C., Michel, C. M., and Caroni, P. (2016). PV plasticity sustained through D1/5 dopamine signaling required for long-term memory consolidation. Nat. Neurosci. 19, 454-464. doi: $10.1038 / \mathrm{nn} .4231$

Katzman, A., Khodadadi-Jamayran, A., Kapeller-Libermann, D., Ye, X., Tsirigos, A., Heguy, A., et al. (2021). Distinct Transcriptomic Profiles in the Dorsal Hippocampus and Prelimbic Cortex Are Transiently Regulated following Episodic Learning. J. Neurosci. 41, 2601-2614. doi: 10.1523/jneurosci.1557-20. 2021

Kawashima, T., Okuno, H., and Bito, H. (2014). A new era for functional labeling of neurons: activity-dependent promoters have come of age. Front. Neural. Circuits. 23:37. doi: 10.3389/fncir.2014.00037
Kawashima, T., Okuno, H., Nonaka, M., Adachi-Morishima, A., Kyo, N., Okamura, M., et al. (2009). Synaptic activity-responsive element in the Arc/Arg3.1 promoter essential for synapse-to-nucleus signaling in activated neurons. Proc. Natl. Acad. Sci. U. S. A. 106, 316-321. doi: 10.1073/pnas.0806518106

Kim, J. I., Cho, H. Y., Han, J. H., and Kaang, B. K. (2016). Which Neurons Will Be the Engram - Activated Neurons and/or More Excitable Neurons. Exp. Neurobiol. 25, 55-63. doi: 10.5607/en.2016.25.2.55

Lacar, B., Linker, S. B., Jaeger, B. N., Krishnaswami, S. R., Barron, J. J., Kelder, M. J. E., et al. (2016). Nuclear RNA-seq of single neurons reveals molecular signatures of activation. Nat. Commun. 19:11022.

Lai, C. S., Franke, T. F., and Gan, W. B. (2012). Opposite effects of fear conditioning and extinction on dendritic spine remodelling. Nature 483, 87-91. doi: 10.1038/ nature 10792

Lamsa, K., and Lau, P. (2019). Long-term plasticity of hippocampal interneurons during in vivo memory processes. Curr. Opin. Neurobiol. 54, 20-27. doi: 10. 1016/j.conb.2018.08.006

Le Bé, J. V., and Markram, H. (2006). Spontaneous and evoked synaptic rewiring in the neonatal neocortex. Proc. Natl. Acad. Sci. U. S. A. 103, 13214-13219. doi: 10.1073/pnas.0604691103

Lee, K., Holley, S. M., Shobe, J. L., Chong, N. C., Cepeda, C., Levine, M. S., et al. (2018). Parvalbumin Interneurons Modulate Striatal Output and Enhance Performance during Associative Learning. Neuron 99:239. doi: 10.1016/j. neuron.2018.06.034

Lein, E., Borm, L. E., and Linnarsson, S. (2017). The promise of spatial transcriptomics for neuroscience in the era of molecular cell typing. Science 358, 64-69. doi: 10.1126/science.aan6827

Letzkus, J. J., Wolff, S. B., and Lüthi, A. (2015). Disinhibition, a Circuit Mechanism for Associative Learning and Memory. Neuron 88, 264-276. doi: 10.1016/j. neuron.2015.09.024

Liao, D., Hessler, N. A., and Malinow, R. (1995). Activation of postsynaptically silent synapses during pairing-induced LTP in CA1 region of hippocampal slice. Nature 375, 400-404. doi: 10.1038/375400a0

Lin, Y., Bloodgood, B. L., Hauser, J. L., Lapan, A. D., Koon, A. C., Kim, T. K., et al. (2008). Activity-dependent regulation of inhibitory synapse development by Npas4. Nature 455, 1198-1204. doi: 10.1038/nature07319

Lisman, J., Cooper, K., Sehgal, M., and Silva, A. J. (2018). Memory formation depends on both synapse-specific modifications of synaptic strength and cellspecific increases in excitability. Nat. Neurosci. 21, 309-314. doi: 10.1038/ s41593-018-0076-6

Liu, X., Ramirez, S., Pang, P. T., Puryear, C. B., Govindarajan, A., Deisseroth, K., et al. (2012). Optogenetic stimulation of a hippocampal engram activates fear memory recall. Nature 484, 381-385. doi: 10.1038/nature11028

Loebrich, S., and Nedivi, E. (2009). The function of activity-regulated genes in the nervous system. Physiol. Rev. 89, 1079-1103. doi: 10.1152/physrev.00013.2009

Lopez, J., Gamache, K., Schneider, R., and Nader, K. (2015). Memory retrieval requires ongoing protein synthesis and NMDA receptor activity-mediated AMPA receptor trafficking. J. Neurosci. 35, 2465-2475. doi: 10.1523/jneurosci. 0735- 14.2015

Lourenço, J., Pacioni, S., Rebola, N., van Woerden, G. M., Marinelli, S., DiGregorio, D., et al. (2014). Non-associative potentiation of perisomatic inhibition alters the temporal coding of neocortical layer 5 pyramidal neurons. PLoS Biol. 12:e1001903. doi: 10.1371/journal.pbio.1001903

Lovett-Barron, M., Kaifosh, P., Kheirbek, M. A., Danielson, N., Zaremba, J. D., Reardon, T. R., et al. (2014). Dendritic inhibition in the hippocampus supports fear learning. Science 343, 857-863. doi: 10.1126/science.1247485

Lucas, E. K., and Clem, R. L. (2018). GABAergic interneurons: The orchestra or the conductor in fear learning and memory. Brain Res. Bull. 141, 13-19. doi: 10.1016/j.brainresbull.2017.11.016

Macosko, E. Z., Basu, A., Satija, R., Nemesh, J., Shekhar, K., Goldman, M., et al. (2015). Highly Parallel Genome-wide Expression Profiling of Individual Cells Using Nanoliter Droplets. Cell 161, 1202-1214. doi: 10.1016/j.cell.2015.05.002

Mardinly, A. R., Spiegel, I., Patrizi, A., Centofante, E., Bazinet, J. E., Tzeng, C. P., et al. (2016). Sensory experience regulates cortical inhibition by inducing IGF1 in VIP neurons. Nature 531, 371-375. doi: 10.1038/nature17187

Marinelli, S., Pacioni, S., Cannich, A., Marsicano, G., and Bacci, A. (2009). Self-modulation of neocortical pyramidal neurons by endocannabinoids. Nat. Neurosci. 12, 1488-1490. doi: 10.1038/nn.2430 
Matsuo, N., Reijmers, L., and Mayford, M. (2008). Spine-type-specific recruitment of newly synthesized AMPA receptors with learning. Science 319, 1104-1107. doi: $10.1126 /$ science. 1149967

Matsuzaki, M., Honkura, N., Ellis-Davies, G. C., and Kasai, H. (2004). Structural basis of long-term potentiation in single dendritic spines. Nature 429, 761-766. doi: 10.1038/nature02617

McEchron, M. D., and Disterhoft, J. F. (1997). Sequence of single neuron changes in CA1 hippocampus of rabbits during acquisition of trace eyeblink conditioned responses. J. Neurophysiol. 78, 1030-1044. doi: 10.1152/jn.1997.78.2.1030

Mendez, P., Stefanelli, T., Flores, C. E., Muller, D., and Lüscher, C. (2018). Homeostatic Plasticity in the Hippocampus Facilitates Memory Extinction. Cell Rep. 22, 1451-1461. doi: 10.1016/j.celrep.2018.01.025

Minatohara, K., Akiyoshi, M., and Okuno, H. (2016). Role of Immediate-Early Genes in Synaptic Plasticity and Neuronal Ensembles Underlying the Memory Trace. Front. Mol. Neurosci. 5:78. doi: 10.3389/fnmol.2015.00078

Moczulska, K. E., Tinter-Thiede, J., Peter, M., Ushakova, L., Wernle, T., Bathellier, B., et al. (2013). Dynamics of dendritic spines in the mouse auditory cortex during memory formation and memory recall. Proc. Natl. Acad. Sci. U. S. A. 110, 18315-18320. doi: 10.1073/pnas.1312508110

Mongillo, G., Rumpel, S., and Loewenstein, Y. (2018). Inhibitory connectivity defines the realm of excitatory plasticity. Nat. Neurosci. 21, 1463-1470. doi: 10.1038/s41593-018-0226-x

Morris, R. G. (2013). NMDA receptors and memory encoding. Neuropharmacology 74, 32-40. doi: 10.1016/j.neuropharm.2013.04.014

Morris, R. G., Moser, E. I., Riedel, G., Martin, S. J., Sandin, J., Day, M., et al. (2003). Elements of a neurobiological theory of the hippocampus: the role of activitydependent synaptic plasticity in memory. Philos. Trans. R. Soc. Lond. B Biol. Sci. 358, 773-786. doi: 10.1098/rstb.2002.1264

Morrison, D. J., Rashid, A. J., Yiu, A. P., Yan, C., Frankland, P. W., and Josselyn, S. A. (2016). Parvalbumin interneurons constrain the size of the lateral amygdala engram. Neurobiol. Learn. Mem. 135, 91-99. doi: 10.1016/j.nlm.2016. 07.007

Nabavi, S., Fox, R., Proulx, C. D., Lin, J. Y., Tsien, R. Y., and Malinow, R. (2014). Engineering a memory with LTD and LTP. Nature 511, 348-352. doi: 10.1038/ nature 13294

Nguyen, P. T., Dorman, L. C., Pan, S., Vainchtein, I. D., Han, R. T., Nakao-Inoue, H., et al. (2020). Microglial Remodeling of the Extracellular Matrix Promotes Synapse Plasticity. Cell 182, 388-403.e15. doi: 10.1016/j.cell.2020.05.050

Nguyen, P. V., Abel, T., and Kandel, E. R. (1994). Requirement of a critical period of transcription for induction of a late phase of LTP. Science 265, 1104-1107. doi: 10.1126/science.8066450

Nikolaienko, O., Patil, S., Eriksen, M. S., and Bramham, C. R. (2018). Arc protein: a flexible hub for synaptic plasticity and cognition. Semin. Cell. Dev. Biol. 77, 33-42. doi: 10.1016/j.semcdb.2017.09.006

Nonaka, A., Toyoda, T., Miura, Y., Hitora-Imamura, N., Naka, M., Eguchi, M., et al. (2014). Synaptic plasticity associated with a memory engram in the basolateral amygdala. J. Neurosci. 34, 9305-9309. doi: 10.1523/jneurosci.4233-13. 2014

Ognjanovski, N., Schaeffer, S., Wu, J., Mofakham, S., Maruyama, D., Zochowski, M., et al. (2017). Parvalbumin-expressing interneurons coordinate hippocampal network dynamics required for memory consolidation. Nat. Commun. 8:15039.

Oh, M. M., and Disterhoft, J. F. (2015). Increased Excitability of Both Principal Neurons and Interneurons during Associative Learning. Neuroscientist 21, 372-384. doi: 10.1177/1073858414537382

Pan, S., Mayoral, S. R., Choi, H. S., Chan, J. R., and Kheirbek, M. A. (2020). Preservation of a remote fear memory requires new myelin formation. Nat. Neurosci. 23, 487-499. doi: 10.1038/s41593-019-0582-1

Park, S., Kramer, E. E., Mercaldo, V., Rashid, A. J., Insel, N., Frankland, P. W., et al. (2016). Neuronal Allocation to a Hippocampal Engram. Neuropsychopharmacology 41, 2987-2993. doi: 10.1038/npp.2016.73

Parkhurst, C. N., Yang, G., Ninan, I., Savas, J. N., Yates, J. R., Lafaille, J. J., et al. (2013). Microglia promote learning-dependent synapse formation through brain-derived neurotrophic factor. Cell 155, 1596-1609. doi: 10.1016/j.cell. 2013.11.030

Pearce, K., Cai, D., Roberts, A. C., and Glanzman, D. L. (2017). Role of protein synthesis and DNA methylation in the consolidation and maintenance of long-term memory in Aplysia. Elife 6:e18299.
Perry, S., Kiragasi, B., Dickman, D., and Ray, A. (2017). The Role of Histone Deacetylase 6 in Synaptic Plasticity and Memory. Cell Rep. 18, 1337-1345. doi: 10.1016/j.celrep.2017.01.028

Pi, H. J., Hangya, B., Kvitsiani, D., Sanders, J. I., Huang, Z. J., and Kepecs, A. (2013). Cortical interneurons that specialize in disinhibitory control. Nature 503, 521-524. doi: 10.1038/nature12676

Pintchovski, S. A., Peebles, C. L., Kim, H. J., Verdin, E., and Finkbeiner, S. (2009). The serum response factor and a putative novel transcription factor regulate expression of the immediate-early gene Arc/Arg3.1 in neurons. J. Neurosci. 29, 1525-1537. doi: 10.1523/jneurosci.5575-08.2009

Ploski, J. E., Monsey, M. S., Nguyen, T., DiLeone, R. J., and Schafe, G. E. (2011). The neuronal PAS domain protein 4 (Npas4) is required for new and reactivated fear memories. PLoS One 6:e23760. doi: 10.1371/journal.pone.0023760

Poo, M. M., Pignatelli, M., Ryan, T. J., Tonegawa, S., Bonhoeffer, T., Martin, K. C., et al. (2016). What is memory? BMC Biol. 14:40. doi: 10.1186/s12915-0160261-6

Pulimood, N. S., Rodrigues, W. D. S., Junior, Atkinson, D. A., Mooney, S. M., and Medina, A. E. (2017). The Role of CREB, SRF, and MEF2 in Activity-Dependent Neuronal Plasticity in the Visual Cortex. J. Neurosci. 37, 6628-6637. doi: 10.1523/jneurosci.0766-17.2017

Ramirez, S., Liu, X., Lin, P. A., Suh, J., Pignatelli, M., Redondo, R. L., et al. (2013). Creating a false memory in the hippocampus. Science 341, 387-391. doi: 10.1126/science.1239073

Rao-Ruiz, P., Couey, J. J., Marcelo, I. M., Bouwkamp, C. G., Slump, D. E., Matos, M. R., et al. (2019). Engram-specific transcriptome profiling of contextual memory consolidation. Nat. Commun. 10:2232.

Reijmers, L. G., Perkins, B. L., Matsuo, N., and Mayford, M. (2007). Localization of a stable neural correlate of associative memory. Science 317, 1230-1233. doi: 10.1126/science.1143839

Roberts, T. F., Tschida, K. A., Klein, M. E., and Mooney, R. (2010). Rapid spine stabilization and synaptic enhancement at the onset of behavioural learning. Nature 463, 948-952. doi: 10.1038/nature08759

Roy, D. S., Arons, A., Mitchell, T. I., Pignatelli, M., Ryan, T. J., and Tonegawa, S. (2016). Memory retrieval by activating engram cells in mouse models of early Alzheimer's disease. Nature 531, 508-512. doi: 10.1038/nature17172

Roy, D. S., Muralidhar, S., Smith, L. M., and Tonegawa, S. (2017). Silent memory engrams as the basis for retrograde amnesia. Proc. Natl. Acad. Sci. U. S. A. 114, E9972-E9979.

Ruediger, S., Vittori, C., Bednarek, E., Genoud, C., Strata, P., Sacchetti, B., et al. (2011). Learning-related feedforward inhibitory connectivity growth required for memory precision. Nature 473, 514-518. doi: 10.1038/nature09946

Ryan, T. J., Roy, D. S., Pignatelli, M., Arons, A., and Tonegawa, S. (2015). Memory. Engram cells retain memory under retrograde amnesia. Science 348, 1007-1013. doi: 10.1126/science.aaa5542

Saha, R. N., Wissink, E. M., Bailey, E. R., Zhao, M., Fargo, D. C., Hwang, J.-Y., et al. (2011). Rapid activity-induced transcription of Arc and other IEGs relies on poised RNA polymerase II. Nat. Neurosci. 14, 848-856. doi: 10.1038/nn.2839

Sanders, J., Cowansage, K., Baumgärtel, K., and Mayford, M. (2012). Elimination of dendritic spines with long-term memory is specific to active circuits. J. Neurosci. 32, 12570-12578. doi: 10.1523/jneurosci.1131-12.2012

Sanz, E., Yang, L., Su, T., Morris, D. R., McKnight, G. S., and Amieux, P. S. (2009). Cell-type-specific isolation of ribosome-associated mRNA from complex tissues. Proc. Natl. Acad. Sci, U. S. A. 106, 13939-13944. doi: 10.1073/ pnas.0907143106

Sauvage, M., Kitsukawa, T., and Atucha, E. (2019). Single-cell memory trace imaging with immediate-early genes. J. Neurosci. Methods 1:108368. doi: 10. 1016/j.jneumeth.2019.108368

Schacter, D. L. (2001). Forgotten Ideas, Neglected Pioneers: Richard Semon and the Story of Memory. Philadelphia: Psychology Press.

Schilling, K., Luk, D., Morgan, J. I., and Curran, T. (1991). Regulation of a foslacZ fusion gene: a paradigm for quantitative analysis of stimulus-transcription coupling. Proc. Natl. Acad. Sci. U. S. A. 1 88, 5665-5669. doi: 10.1073/pnas.88. 13.5665

Sehgal, M., Zhou, M., Lavi, A., Huang, S., Zhou, Y., and Silva, A. J. (2018). Memory allocation mechanisms underlie memory linking across time. Neurobiol. Learn. Memory 153, 21-25. doi: 10.1016/j.nlm.2018.02.021

Sherwood, L. (2015). Human Physiology: From Cells to Systems. Boston, MA: Cengage Learning, 157-162. 
Sørensen, A. T., Cooper, Y. A., Baratta, M. V., Weng, F. J., Zhang, Y., Ramamoorthi, K., et al. (2016). A robust activity marking system for exploring active neuronal ensembles. Elife. 23:e13918.

Spiegel, I., Mardinly, A. R., Gabel, H. W., Bazinet, J. E., Couch, C. H., Tzeng, C. P., et al. (2014). Npas4 regulates excitatory-inhibitory balance within neural circuits through cell-type-specific gene programs. Cell 157, 1216-1229. doi: 10.1016/j.cell.2014.03.058

Sprekeler, H. (2017). Functional consequences of inhibitory plasticity: homeostasis, the excitation-inhibition balance and beyond. Curr. Opin. Neurobiol. 43, 198203. doi: 10.1016/j.conb.2017.03.014

Steadman, P. E., Xia, F., Ahmed, M., Mocle, A. J., Penning, A. R. A., Geraghty, A. C., et al. (2020). Disruption of Oligodendrogenesis Impairs Memory Consolidation in Adult Mice. Neuron 105, 150-164.e6. doi: 10.1016/j.neuron.2019.10.013

Stefanelli, T., Bertollini, C., Lüscher, C., Muller, D., and Mendez, P. (2016). Hippocampal Somatostatin Interneurons Control the Size of Neuronal Memory Ensembles. Neuron 89, 1074-1085. doi: 10.1016/j.neuron.2016.01.024

Su, Y., Shin, J., Zhong, C., Wang, S., Roychowdhury, P., Lim, J., et al. (2017). Neuronal activity modifies the chromatin accessibility landscape in the adult brain. Nat. Neurosci. 20, 476-483. doi: 10.1038/nn.4494

Sun, X., Bernstein, M. J., Meng, M., Rao, S., Sørensen, A. T., Yao, L., et al. (2020). Functionally Distinct Neuronal Ensembles within the Memory Engram. Cell 181, 410-423.e17.

Tonegawa, S., Morrissey, M. D., and Kitamura, T. (2018). The role of engram cells in the systems consolidation of memory. Nat. Rev. Neurosci. 19, 485-498. doi: 10.1038/s41583-018-0031-2

Tonegawa, S., Pignatelli, M., Roy, D. S., and Ryan, T. J. (2015). Memory engram storage and retrieval. Curr. Opin. Neurobiol. 35, 101-109. doi: 10.1016/j.conb. 2015.07.009

Turrigiano, G. (2012). Homeostatic synaptic plasticity: local and global mechanisms for stabilizing neuronal function. Cold Spring Harb. Perspect. Biol. 4:a005736. doi: 10.1101/cshperspect.a005736

Tyssowski, K. M., DeStefino, N. R., Cho, J. H., Dunn, C. J., Poston, R. G., Carty, C. E., et al. (2018). Different Neuronal Activity Patterns Induce Different Gene Expression Programs. Neuron 98, 530-546.e11. doi: 10.1016/j.neuron.2018. 04.001

Vallentin, D., Kosche, G., Lipkind, D., and Long, M. A. (2016). Neural circuits. Inhibition protects acquired song segments during vocal learning in zebra finches. Science 351, 267-271. doi: 10.1126/science.aad3023

Vazdarjanova, A., Ramirez-Amaya, V., Insel, N., Plummer, T. K., Rosi, S., Chowdhury, S., et al. (2006). Spatial exploration induces ARC, a plasticityrelated immediate-early gene, only in calcium/calmodulin-dependent protein kinase II-positive principal excitatory and inhibitory neurons of the rat forebrain. J. Comp. Neurol. 498, 317-329. doi: 10.1002/cne.21003

Vogels, T. P., Sprekeler, H., Zenke, F., Clopath, C., and Gerstner, W. (2011). Inhibitory plasticity balances excitation and inhibition in sensory pathways and memory networks. Science 334, 1569-1573. doi: 10.1126/science.1211095

Waltereit, R., Dammermann, B., Wulff, P., Scafidi, J., Staubli, U., Kauselmann, G., et al. (2001). Arg3.1/Arc mRNA induction by $\mathrm{Ca} 2+$ and cAMP requires protein kinase $\mathrm{A}$ and mitogen-activated protein kinase/extracellular regulated kinase activation. J. Neurosci. 21, 5484-5493. doi: 10.1523/jneurosci.21-1505484.2001

Wang, X., Allen, W. E., Wright, M. A., Sylwestrak, E. L., Samusik, N., Vesuna, S., et al. (2018). Three-dimensional intact-tissue se-quencing of single-cell transcriptional states. Science 361:eaat5691. doi: 10.1126/science.aat5691

Wolff, S. B., Gründemann, J., Tovote, P., Krabbe, S., Jacobson, G. A., Müller, C., et al. (2014). Amygdala interneuron subtypes control fear learning through disinhibition. Nature 509, 453-458. doi: 10.1038/nature13258

Wu, Y. E., Pan, L., Zuo, Y., Li, X., and Hong, W. (2017). Detecting Activated Cell Populations Using Single-Cell RNA-Seq. Neuron 96, 313-329. doi: 10.1016/j. neuron.2017.09.026

Xia, F., Richards, B. A., Tran, M. M., Josselyn, S. A., Takehara-Nishiuchi, K., and Frankland, P. W. (2017). Parvalbumin-positive interneurons mediate neocortical-hippocampal interactions that are necessary for memory consolidation. Elife 6:e27868.

Xu, H., Liu, L., Tian, Y., Wang, J., Li, J., Zheng, J., et al. (2019). A Disinhibitory Microcircuit Mediates Conditioned Social Fear in the Prefrontal Cortex. Neuron 102, 668-682.e5.

Yang, G., Pan, F., and Gan, W. B. (2009). Stably maintained dendritic spines are associated with lifelong memories. Nature 462, 920-924. doi: 10.1038/ nature 08577

Yap, E. L., and Greenberg, M. E. (2018). Activity-Regulated Transcription: Bridging the Gap between Neural Activity and Behavior. Neuron 100, 330-348. doi: 10.1016/j.neuron.2018.10.013

Yap, E. L., Pettit, N. L., Davis, C. P., Nagy, M. A., Harmin, D. A., Golden, E., et al. (2021). Bidirectional perisomatic inhibitory plasticity of a Fos neuronal network. Nature 590, 115-121. doi: 10.1038/s41586-020-3031-0

Yiu, A. P., Mercaldo, V., Yan, C., Richards, B., Rashid, A. J., Hsiang, H. L., et al. (2014). Neurons are recruited to a memory trace based on relative neuronal excitability immediately before training. Neuron $83,722-735$. doi: 10.1016/j. neuron.2014.07.017

Zhou, Y., Won, J., Karlsson, M. G., Zhou, M., Rogerson, T., and Balaji, J. (2009). CREB regulates excitability and the allocation of memory to subsets of neurons in the amygdala. Nat. Neurosci. 12, 1438-1443. doi: 10.1038/nn. 2405

Zhou, Z., Okamoto, K., Onodera, J., Hiragi, T., Andoh, M., and Ikawa, M. (2021). Astrocytic cAMP modulates memory via synaptic plasticity. Proc. Natl. Acad. Sci. U. S. A. 118:e2016584118.

Conflict of Interest: The authors declare that the research was conducted in the absence of any commercial or financial relationships that could be construed as a potential conflict of interest.

Copyright (c) 2021 Giorgi and Marinelli. This is an open-access article distributed under the terms of the Creative Commons Attribution License (CC BY). The use, distribution or reproduction in other forums is permitted, provided the original author(s) and the copyright owner(s) are credited and that the original publication in this journal is cited, in accordance with accepted academic practice. No use, distribution or reproduction is permitted which does not comply with these terms. 